\title{
NUCLEAR MASS INVENTORY, PHOTON DOSE RATE AND THERMAL DECAY HEAT OF SPENT RESEARCH REACTOR FUEL ASSEMBLIES
}

RECEIVED

R. B. Pond and J. E. Matos

RERTR Program

Argonne National Laboratory

Argonne, IL 60439-4841 USA
AUG 121996

OSTI

May 1996

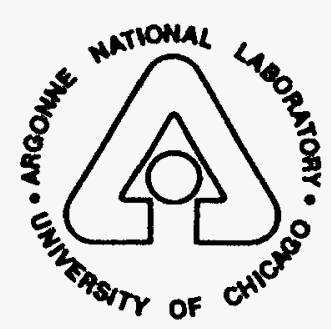

ARGONNE NATIONAL LABORATORY, ARGONNE, ILLINOIS

Operated by The University of Chicago

for the United States Department of Energy

under Contract No. W-31-109-Eng-38. 
Argonne National Laboratory, with facilities in the states of Illinois and Idaho, is owned by the United States government, and operated by The University of Chicago under the provisions of a contract with the Department of Energy.

\section{DISCLAIMER}

This report was prepared as an account of work sponsored by an agency of the United States Government. Neither the United States Government nor any agency thereof, nor any of their employees, makes any warranty, expressed or implied, or assumes any legal liability or responsibility for the accuracy, completeness, or usefulness of any information, apparatus, product, or process disclosed, or represents that its use would not infringe privately owned rights. Reference herein to any specific commercial product, process, or service by trade name, trademark, manufacturer, or otherwise, does not necessarily constitute or imply its endorsement, recommendation, or favoring by the United States Government or any agency thereof. The views and opinions of authors expressed herein do not necessarily state or reflect those of the United States Government or any agency thereot.

Available from the

Reduced Enrichment for Research and Test Reactor (RERTR) Program

Argonne National Laboraton'

9700 South Cass Avenue

Argonne, IL 60439-4841 USA 


\title{
NUCLEAR MASS INVENTORY, PHOTON DOSE RATE AND THERMAL DECAY HEAT OF SPENT RESEARCH REACTOR FUEL ASSEMBLIES
}

\author{
R. B. Pond and J. E. Matos \\ RERTR Program \\ Argonne National Laboratory \\ Argonne, IL 60439-4841 USA
}

May 1996

\section{DISCLAIMER}

This report was prepared as an account of work sponsored by an agency of the United States Government. Neither the United States Government nor any agency thereof, nor any of their employees, makes any warranty, express or implied, or assumes any legal liability or responsibility for the accuracy, completeness, or usefulness of any information, apparatus, product, or process disclosed, or represents that its use would not infringe privately owned rights. Reference herein to any specific commercial product, process, or service by trade name, trademark, manufacturer, or otherwise does not necessarily constitute or imply its endorsement, recommendation, or favoring by the United States Government or any agency thereof. The views and opinions of authors expressed herein do not necessarily state or reflect those of the United States Government or any agency thereof.

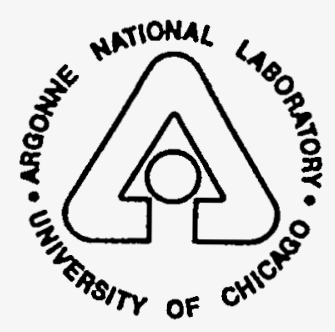

\section{ARGONNE NATIONAL LABORATORY, ARGONNE, ILLINOIS}

\author{
Operated by The University of Chicago \\ for the United States Department of Energy \\ under Contract No. W-31-109-Eng-38.
}




\section{DISCLAIMER}

Portions of this document may be illegible in electronic image products. Images are produced from the best available original document. 


\section{NUCLEAR MASS INVENTORY, PHOTON DOSE RATE AND THERMAL DECAY HEAT} OF SPENT RESEARCH REACTOR FUEL ASSEMBLIES

\section{TABLE OF CONTENTS}

Page

Summary

Introduction

Nuclear Mass Inventory

Photon Dose Rate

Thermal Decay Heat

Conclusions

References

Appendix A: MTR Model Mass Inventory Sensitivity

Appendix B: U-234 And U-236 Mass Inventory Sensitivity 


\title{
NUCLEAR MASS INVENTORY, PHOTON DOSE RATE AND THERMAL DECAY HEAT \\ OF SPENT RESEARCH REACTOR FUEL ASSEMBLIES
}

\author{
R. B. Pond and J. E. Matos \\ Argonne National Laboratory \\ Argonne, II.
}

\begin{abstract}
SUMMARY
This document has been prepared to assist research reactor operators possessing spent fuel containing enriched uranium of United States origin to prepare part of the documentation necessary to ship this fuel to the United States. Data are included on the nuclear mass inventory, photon dose rate, and thermal decay heat of spent research reactor fuel assemblies.

Isotopic masses of $\mathrm{U}, \mathrm{Np}, \mathrm{Pu}$ and $\mathrm{Am}$ that are present in spent research reactor fuel are estimated for MTR, TRIGA and DIDO fuel assembly types. The isotopic masses of each fuel assembly type are given as functions of U-235 burnup in the spent fuel, and of initial U-235 enrichment and U-235 mass in the fuel assembly.
\end{abstract}

Photon dose rates of spent MTR, TRIGA and DIDO-type fuel assemblies are estimated for fuel assemblies with up to $80 \%$ U-235 burnup and specific power densities between 0.089 and $2.857 \mathrm{MW} / \mathrm{kg}^{235} \mathrm{U}$, and for fission product decay times of up to 20 years.

Thermal decay heat loads are estimated for spent fuel based upon the fuel assembly irradiation history (average assembly power vs. elapsed time) and the spent fuel cooling time.

\section{INTRODUCTION}

As part of the Department of Energy's spent nuclear fuel acceptance criteria, the mass of uranium and transuranic elements in spent research reactor fuel must be specified. These data are, however, not always known or readily determined. It is the purpose of this report to provide estimates of these data for some of the more common research reactor fuel assembly types. The specific types considered here are MTR, TRIGA and DIDO fuel assemblies.

The degree of physical protection given to spent fuel assemblies is largely dependent upon the photon dose rate of the spent fuel material. These data also, are not always known or readily determined. Because of a self-protecting dose rate level of radiation (dose rate greater than $100 \mathrm{rem} / \mathrm{h}$ at $1 \mathrm{~m}$ in air), it is important to know the dose 
rate of spent fuel assemblies at all time. Estimates of the photon dose rate for spent MTR, TRIGA and DIDO-type fuel assemblies are given in this report.

For safe spent fuel assembly containment, the thermal heat load generated by the decay of fission products in spent fuel material is an important consideration. This heat load can be estimated by a simple analytical expression that is given in this report.

\section{NUCLEAR MASS INVENTORY}

The mass inventory of the heavy metals in research reactor fuels has been calculated using the WIMS code ${ }^{1}$ for unit-cell models of MTR, TRIGA and DIDO fuel assembly types. Models of each fuel assembly type were neutronically burned for a length of time corresponding to typical fuel-cycle lengths and U-235 burnup ${ }^{2}$. Table 1 summarizes the fuel assembly models for which mass inventory calculations were made.

Table 1. Fuel Assembly Models

\begin{tabular}{|c|c|c|c|}
\hline Assembly Type & U-235 Burnup, \% & U-235 Enrichment, \% & U-235 Mass, $g$ \\
\hline MTR & $5,10,20,30,40,50,60,70,80$ & 93 & 100200300400 \\
(19 fuel plates) & & 45 & 200300400 \\
& & 19.75 & 100200300400500 \\
\hline TRIGA & $5,10,15,20,25,30,35$ & $70(8.5 w \%$ U) & 133 \\
(single rod) & & $20(20 w t \%$ U) & 98 \\
& & $20(12 w t \% U)$ & 54 \\
& & $20(8.5 w \% U)$ & 38 \\
\hline TRIGA & $10,20,30,40,50,60$ & $93.1(10 w t \% U)$ & 41.4 \\
(25 rod cluster) & & $19.7(45 w t \% U)$ & 53.6 \\
\hline DIDO & $10,20,30,40,50,60$ & 93 & 150 \\
(4 fuel tubes) & & 80 & 150 \\
& & 60 & 150 \\
& & 20 & 200 \\
\hline
\end{tabular}

Mass inventory calculations for MTR models were made for assemblies with up to $80 \%$ U-235 burnup, for 93,45 and $19.75 \%$ U-235 enrichments, and for initial U-235 masses of 100 to $500 \mathrm{~g}$. The specific MTR model was for a 19-fuel plate assembly. (Supplemental mass inventory calculations, shown in Appendix A, indicate that the MTR model in not a strong function of the number of fuel plates or the specific fuel-cladcoolant geometry.)

Similar calculations were made for two TRIGA assembly types - a single rod model and a 25-rod cluster model. The maximum U-235 burnup in these models were respectively, 35 and $60 \%$. There were four fuel types for the single rod model and two fuel types for the cluster model.

For DIDO fuel assembly types, mass inventory calculations were made for a 4fuel tube model with up to $60 \%$ U-235 burnup, and for four fuel enrichments and assembly masses. 
The results of the mass inventory calculations are shown in the following tables:

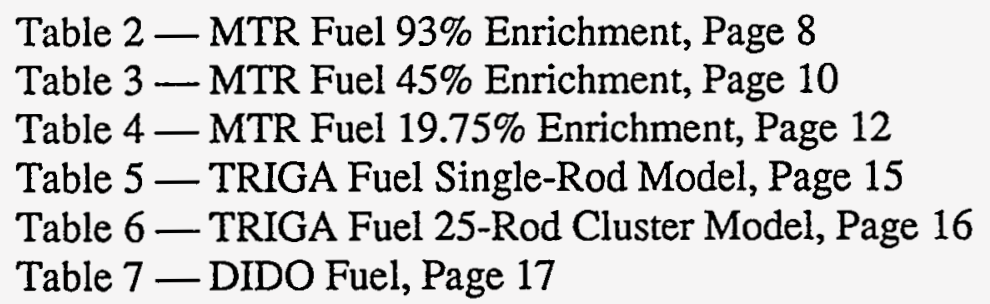

The tables show the isotopic masses of $\mathrm{U}, \mathrm{Np}, \mathrm{Pu}$ and Am that are present in spent fuel as functions of the fuel assembly U-235 burnup and initial U-235 mass. As will be noted in the tables for most fuel assembly types, the uranium fuel compositions have excluded initial enrichments of U-234 and U-236. In order to account for initial enrichments of U234 and/or U-236 in the tables, initial U-234 and U-236 masses can be simply added to the spent fuel mass inventory. (See Appendix B for an assessment of the effect of initial enrichments of U-234 and U-236 upon the overall mass inventory of $\mathrm{U}, \mathrm{Np}, \mathrm{Pu}$ and $\mathrm{Am}$ in spent fuel.) Within the uncertainty of the calculations, the results in Tables 2-7 can be used to estimate the spent fuel mass inventory in most MTR, TRIGA and DIDO fuel assembly types.

The mass inventories given in Tables 2-7 are at the time of reactor discharge and therefore do not account for decay of Pu-241 to Am-241 for times after discharge. When necessary to estimate mass inventories after discharge, the Pu-241 mass is decreased and the Am-241 mass is increased by an amount $\Delta M=M_{0} \cdot\left(1-e^{-\lambda t}\right)$ where $M_{0}$ is the Pu241 mass at discharge, $\lambda=1.32 \cdot 10^{-4} \mathrm{~d}^{-1}(\mathrm{Pu}-241$ half-life, $14.4 \mathrm{y})$, and $t$ is the time in days after discharge. No mass inventories are given for U-239 (half-life, $23.5 \mathrm{~m}$ ) and Np239 (half life, $2.355 \mathrm{~d}$ ) as they are assumed to decay instantaneously to Pu-239.

\section{PHOTON DOSE RATE}

Calculated dose rates for MTR-type fuel assemblies are shown in Table 8. These dose rates are from Ref. 3 and are for fuel assemblies with up to $80 \%$ U-235 burnup, specific power densities between 0.089 and $2.857 \mathrm{MW} / \mathrm{kg}^{235} \mathrm{U}$, and fission product decay times of up to 20 years.

The data in Table 8 are photon dose rates in air that are averaged over a $60-\mathrm{cm}$ long cylindrical surface, located at a radius of $1 \mathrm{~m}$ from the fuel assembly axial center line. For MTR-type fuel assemblies, these average dose rates are independent of the assembly rotational orientation and the number of fuel plates in the assembly. These data also can be interpolated for specific decay time, burnup and assembly power density. In all cases, the dose rates must be multiplied by the mass of U-235 burned in the fuel assembly to estimate the fuel assembly dose rate. The mass of U-235 burned per fuel assembly that is necessary for an unshielded, $100 \mathrm{rem} / \mathrm{h}$ self-protecting dose rate at $1 \mathrm{~m}$, is shown in Fig. 1. 
Additional analyses have shown that the photon dose rates of MTR, TRIGA and DIDO-type fuel assemblies are similar, given the same fuel assembly characteristics of U-235 burnup, fission product decay time, and specific fuel assembly power density. The average dose rates at $1 \mathrm{~m}$ in air for TRIGA (25-rod) and DIDO (4-tube) fuel assemblies are respectively, 1.04 and 1.05 times the dose rates given in Table 8 for MTR fuel assemblies. The dose rates of all three fuel assembly types are for fuel assembly models (nominally $8 \mathrm{~cm}$ by $8 \mathrm{~cm}$ by $60 \mathrm{~cm}$ ) containing spent fuel in the form of either rods (TRIGA fuel), annuli (DIDO fuel) or plates (MTR fuel). The small difference in the dose rates are due to the different shielding effects of the fuel elements in the fuel assemblies.

Table 8. Photon Dose Rates At $1 \mathrm{M}$ In Air, rem/h per $\mathrm{g}^{25} \mathrm{U}$ burned

\begin{tabular}{|c|c|c|c|c|c|c|c|}
\hline $\begin{array}{c}\text { Decay } \\
\text { Time, }\end{array}$ & $\begin{array}{c}\text { Burnup, } \\
\%{ }^{25} \mathrm{U}\end{array}$ & \multicolumn{6}{|c|}{ Assembly Power Density, MW/kg } \\
\hline & & 2.857 & 1.429 & 0.714 & 0.357 & 0.179 & 0.089 \\
\hline 2 & $1 \%$ & $1.84+0$ & $1.84+0$ & $1.83+0$ & $1.80+0$ & $1.77+0$ & $1.70+0$ \\
3 & & $1.13+0$ & $1.13+0$ & $1.13+0$ & $1.13+0$ & $1.11+0$ & $1.11+0$ \\
4 & & $9.01-1$ & $9.01-1$ & $9.01-1$ & $9.01-1$ & $9.01-1$ & $8.92-1$ \\
\hline 2 & $10 \%$ & $1.89+0$ & $1.87+0$ & $1.80+0$ & $1.64+0$ & $1.50+0$ & $1.28+0$ \\
3 & & $1.19+0$ & $1.20+0$ & $1.20+0$ & $1.16+0$ & $1.09+0$ & $9.95-1$ \\
4 & & $9.52-1$ & $9.61-1$ & $9.61-1$ & $9.44-1$ & $9.10-1$ & $8.59-1$ \\
\hline 2 & $20 \%$ & $2.01+0$ & $1.98+0$ & $1.86+0$ & $1.66+0$ & $1.42+0$ & $1.19+0$ \\
3 & & $1.31+0$ & $1.32+0$ & $1.28+0$ & $1.21+0$ & $1.11+0$ & $9.78-1$ \\
4 & & $1.04+0$ & $1.05+0$ & $1.04+0$ & $9.99-1$ & $9.44-1$ & $8.63-1$ \\
5 & & $8.97-1$ & $9.10-1$ & $9.05-1$ & $8.80-1$ & $8.46-1$ & $7.95-1$ \\
10 & & $6.67-1$ & $6.67-1$ & $6.67-1$ & $6.59-1$ & $6.50-1$ & $6.25-1$ \\
15 & & $5.78-1$ & $5.78-1$ & $5.74-1$ & $5.70-1$ & $5.61-1$ & $5.44-1$ \\
20 & & $5.10-1$ & $5.10-1$ & $5.10-1$ & $5.06-1$ & $4.97-1$ & $4.85-1$ \\
\hline 2 & $40 \%$ & $2.40+0$ & $2.30+0$ & $2.09+0$ & $1.82+0$ & $1.52+0$ & $1.21+0$ \\
3 & & $1.62+0$ & $1.60+0$ & $1.53+0$ & $1.39+0$ & $1.22+0$ & $1.02+0$ \\
4 & & $1.27+0$ & $1.27+0$ & $1.22+0$ & $1.14+0$ & $1.03+0$ & $8.99-1$ \\
5 & & $1.07+0$ & $1.07+0$ & $1.04+0$ & $9.90-1$ & $9.20-1$ & $8.12-1$ \\
10 & & $7.03-1$ & $7.03-1$ & $6.95-1$ & $6.80-1$ & $6.55-1$ & $6.10-1$ \\
15 & & $5.87-1$ & $5.84-1$ & $5.80-1$ & $5.70-1$ & $5.53-1$ & $5.23-1$ \\
20 & & $5.14-1$ & $5.12-1$ & $5.08-1$ & $5.02-1$ & $4.87-1$ & $4.59-1$ \\
\hline 2 & $60 \%$ & $2.95+0$ & $2.79+0$ & $2.52+0$ & $2.15+0$ & $1.74+0$ & $1.34+0$ \\
3 & & $2.05+0$ & $2.00+0$ & $1.87+0$ & $1.66+0$ & $1.40+0$ & $1.12+0$ \\
4 & & $1.59+0$ & $1.56+0$ & $1.49+0$ & $1.35+0$ & $1.17+0$ & $9.63-1$ \\
5 & & $1.30+0$ & $1.29+0$ & $1.24+0$ & $1.15+0$ & $1.02+0$ & $8.54-1$ \\
10 & & $7.55-1$ & $7.51-1$ & $7.37-1$ & $7.07-1$ & $6.70-1$ & $6.02-1$ \\
15 & & $5.96-1$ & $5.96-1$ & $5.88-1$ & $5.72-1$ & $5.50-1$ & $5.04-1$ \\
20 & & $5.17-1$ & $5.17-1$ & $5.13-1$ & $4.99-1$ & $4.76-1$ & $4.39-1$ \\
\hline 2 & $80 \%$ & $3.85+0$ & $3.62+0$ & $3.26+0$ & $2.76+0$ & $2.21+0$ & $1.64+0$ \\
3 & & $2.73+0$ & $2.64+0$ & $2.43+0$ & $2.11+0$ & $1.74+0$ & $1.33+0$ \\
4 & & $2.08+0$ & $2.03+0$ & $1.90+0$ & $1.69+0$ & $1.41+0$ & $1.12+0$ \\
5 & & $1.66+0$ & $1.63+0$ & $1.54+0$ & $1.39+0$ & $1.19+0$ & $9.57-1$ \\
10 & & $8.28-1$ & $8.21-1$ & $8.00-1$ & $7.59-1$ & $6.97-1$ & $6.04-1$ \\
15 & & $6.18-1$ & $6.15-1$ & $6.05-1$ & $5.82-1$ & $5.44-1$ & $4.87-1$ \\
20 & & $5.27-1$ & $5.20-1$ & $5.13-1$ & $4.97-1$ & $4.66-1$ & $4.20-1$ \\
\hline
\end{tabular}



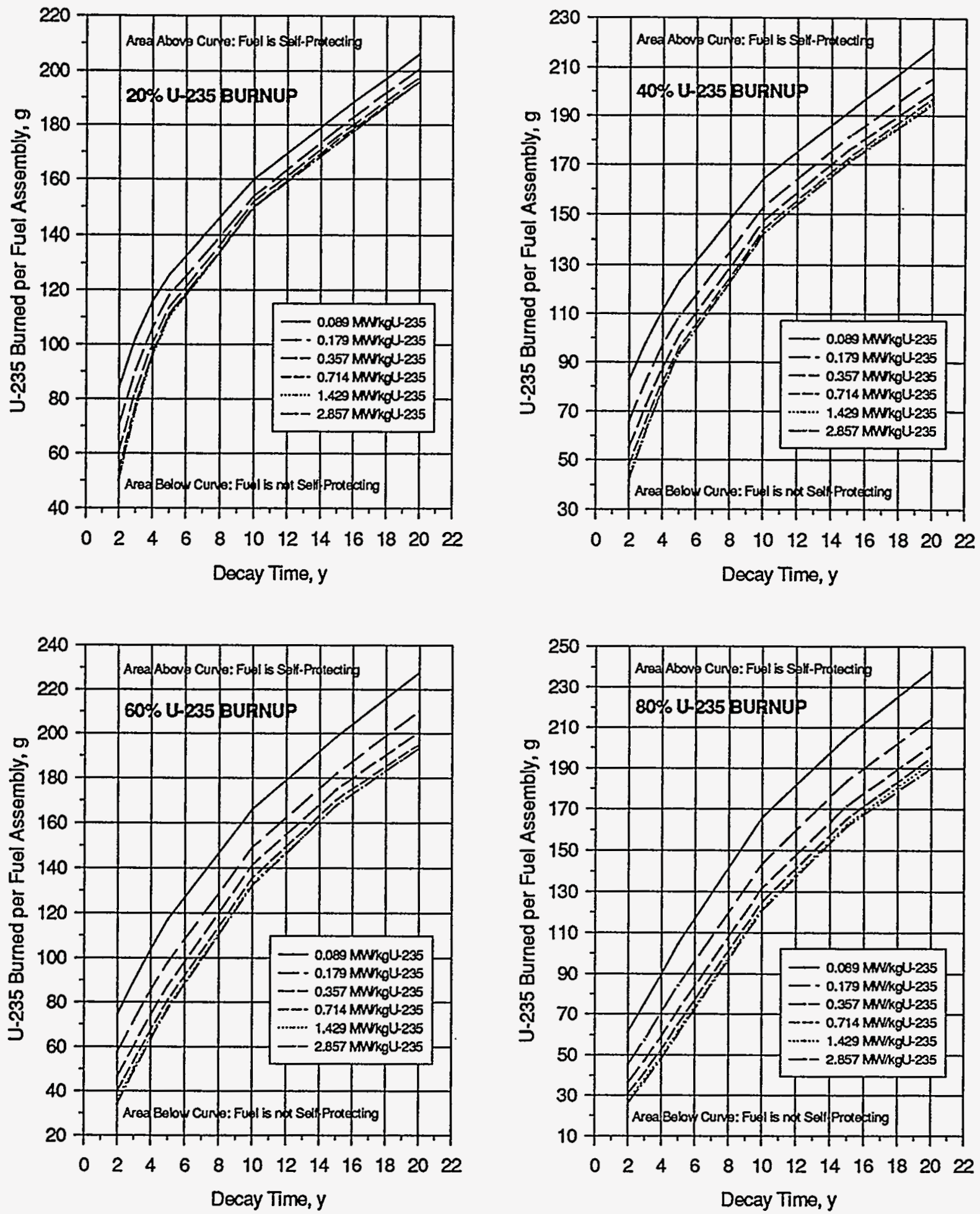

Figure 1. Mass of Burned ${ }^{25} \mathrm{U}$ per Fuel Assembly Necessary for an Unshielded $100 \mathrm{rem} / \mathrm{h}$ Dose Rate at $1 \mathrm{~m}$ for Fuel Assemblles with $20,40,60$ and $80 \%{ }^{23} \mathrm{U}$ Burnup and Power Densities from 0.089 to $2.857 \mathrm{MW} / \mathrm{kg}^{23} \mathrm{U}$ 
The heat load from decaying fission products in a fuel assembly is proportional to empirical emission rates of beta and gamma radiation. The rates ${ }^{4}$ per U-235 fission, and as a function of decay time $t_{d}$ in days, are

$$
\begin{aligned}
& \beta\left(t_{d}\right)=1.50 \cdot 10^{-6} \cdot t_{d}^{-1.2} \mathrm{MeV} / \mathrm{s}-\mathrm{f} \\
& \gamma\left(t_{d}\right)=1.67 \cdot 10^{-6} \cdot t_{d}{ }^{-1.2} \mathrm{MeV} / \mathrm{s}-\mathrm{f}
\end{aligned}
$$

These energy rates are roughly equal for $0.4 \mathrm{MeV}$ mean energy beta particles and 0.7 $\mathrm{MeV}$ mean energy gamma-rays.

For a fuel assembly irradiated continuously for $t_{i}$ days at a constant fuel assembly power $(P)$, the heat $(H)$ load power per assembly, $t_{d}$ days after irradiation is

$$
H=6.85 \cdot 10^{-3} \cdot P \cdot\left(t_{d}{ }^{-0.2}-\left(t_{i}+t_{d}\right)^{-0.2}\right) \text { Watts }
$$

This expression for the heat load is the integral of the above energy rates over the irradiation time, assuming $200 \mathrm{MeV}$ per U-235 fission, and for the fuel assembly power in watts. For a low duty-factor fuel assembly irradiation, the power and irradiation time are replaced by an average power and an elapsed time. With $\bar{P} \cdot t_{e}=\sum\left(P \cdot t_{i}\right)$ over all irradiation segments, the heat $(H)$ load power per assembly is

$$
H \equiv 6.85 \cdot 10^{-3} \cdot \bar{P} \cdot\left(t_{d}^{-0.2}-\left(t_{e}+t_{d}\right)^{-0.2}\right) \text { Watts }
$$

where $\bar{P}$ is the average fuel assembly power in watts and $t_{e}$ is the elapsed time in days from the initial through the final irradiation segment.

A convenient estimate for the average power $(\bar{P})$ is

$$
\bar{P}=\left(G / t_{e}\right) / 1.25 \cdot 10^{-6} \text { Watts }
$$

where $G$ is the mass of U-235 burned in the fuel assembly in grams, and the constant is $\mathrm{g}^{235} \mathrm{U}$ burned per $\mathrm{Wd}$.

Fuel assembly decay heat loads calculated with these expressions are expected to be conservative, and within a factor of two or less of measured heat loads. This same conservative heat load estimate also has been found to be true for heat load calculations made with the ORIGEN code 6 . The thermal heat load of a fuel assembly is independent of the fuel assembly type. 


\section{CONCLUSIONS}

Procedures have been developed to estimate the nuclear mass inventory, the photon dose rate and the thermal decay heat of spent research reactor fuel assemblies. The procedures should provide reasonable estimates based upon known fuel assembly parameters.

Isotopic mass inventories of $\mathrm{U}, \mathrm{Np}, \mathrm{Pu}$ and Am are tabulated in Tables 2-7 for MTR, TRIGA and DIDO fuel assembly types; photon dose rates at $1 \mathrm{~m}$ in air are shown in Table 8 for MTR-type fuel assemblies; and an analytical expression is given for the thermal decay heat load of spent uranium fuel. Estimates of TRIGA and DDO fuel assembly dose rates are respectively, factors of 1.04 and 1.05 times the dose rate for MTR-type fuel assemblies with similar spent fuel material characteristics.

\section{REFERENCES}

1. J. R. Deen, W. L. Woodruff and C.I. Costescu, "WIMS-D4M User Manual (Rev. 0)", ANL/RERTR/TM-23, Argonne National Laboratory, Argonne, IL (July 1995).

2. J. E. Matos, "Foreign Research Reactor Irradiated Nuclear Fuel Inventories Containing HEU And LEU Of United States Origin", ANL/RERTR/TM-22, Argonne National Laboratory, Argonne, IL (December 1994).

3. R. B. Pond and J. E. Matos, "Photon Dose Rates From Spent Fuel Assemblies With Relation To Self-Protection (Rev. 1)", ANL/RERTR/TM-25, Argonne National Laboratory, Argonne, IL (February 1996).

4. A. M. Weinberg and E. P. Wigner, "The Physical Theory Of Neutron Chain Reactors", University of Chicago Press, Chicago, IL (1958).

5. S. Glasstone, "Principles Of Nuclear Reactor Engineering", D. Van Nostrand Company, Princeton, NJ (1955).

6. M. J. Bell, "ORIGEN — The ORNL Isotope Generation And Depletion Code," ORNL-4628, Oak Ridge National Laboratory, Oak Ridge, TN (May 1973). 
Table 2. MTR Fuel $93 \%$ Enrichment

\begin{tabular}{|c|c|c|c|c|c|c|c|c|c|c|}
\hline MTR Fuel & $93 \%$ & ment & & 100 & & & & & & \\
\hline $\begin{array}{l}\text { U-235 Burnup, \% } \\
\text { U-235 Bumed, } g\end{array}$ & $\begin{array}{l}0 \\
0\end{array}$ & $\begin{array}{l}5 \\
5\end{array}$ & $\begin{array}{l}10 \\
10 \\
\end{array}$ & $\begin{array}{l}20 \\
20\end{array}$ & $\begin{array}{l}30 \\
30\end{array}$ & $\begin{array}{l}40 \\
40\end{array}$ & $\begin{array}{l}50 \\
50\end{array}$ & $\begin{array}{l}60 \\
60\end{array}$ & $\begin{array}{l}70 \\
70\end{array}$ & $\begin{array}{l}80 \\
80\end{array}$ \\
\hline U-234 & 0 & 0 & 0 & 0 & 0 & 0 & 0 & 0 & 0 & 0 \\
\hline$U-235$ & 100 & 95 & 90 & 80 & 70 & 60 & 50 & 40 & 30 & 20 \\
\hline U-236 & 0 & 1 & 2 & 3 & 5 & 6 & 8 & 9 & 11 & 12 \\
\hline U-238 & 8 & 8 & 8 & 8 & 7 & 7 & 7 & 7 & 7 & 7 \\
\hline $\mathrm{U}$ & 108 & 103 & 99 & 91 & 82 & 74 & 65 & 56 & 48 & 39 \\
\hline Np-237 & 0 & 0.0 & 0.0 & 0.0 & 0.0 & 0.1 & 0.1 & 0.2 & 0.2 & 0.3 \\
\hline $\mathrm{Np}$ & 0 & 0.0 & 0.0 & 0.0 & 0.0 & 0.1 & 0.1 & 0.2 & 0.2 & 0.3 \\
\hline $\mathrm{Pu}-238$ & 0 & 0.0 & 0.0 & 0.0 & 0.0 & 0.0 & 0.0 & 0.0 & 0.0 & 0.0 \\
\hline Pu-239 & 0 & 0.0 & 0.0 & 0.0 & 0.1 & 0.1 & 0.1 & 0.1 & 0.1 & 0.1 \\
\hline Pu-240 & 0 & 0.0 & 0.0 & 0.0 & 0.0 & 0.0 & 0.0 & 0.0 & 0.0 & 0.0 \\
\hline Pu-241 & 0 & 0.0 & 0.0 & 0.0 & 0.0 & 0.0 & 0.0 & 0.0 & 0.0 & 0.0 \\
\hline $\mathrm{Pu}-242$ & 0 & 0.0 & 0.0 & 0.0 & 0.0 & 0.0 & 0.0 & 0.0 & 0.0 & 0.0 \\
\hline $\mathrm{Pu}$ & 0 & 0.0 & 0.0 & 0.0 & 0.1 & 0.1 & 0.1 & 0.1 & 0.2 & 0.2 \\
\hline Am-241 & 0 & 0.0 & 0.0 & 0.0 & 0.0 & 0.0 & 0.0 & 0.0 & 0.0 & 0.0 \\
\hline $\mathrm{Am}$ & 0 & 0.0 & 0.0 & 0.0 & 0.0 & 0.0 & 0.0 & 0.0 & 0.0 & 0.0 \\
\hline MTR Fuel & $93 \%$ & ment & & 200 & & & & & & \\
\hline $\begin{array}{l}\text { U-235 Burnup, \% } \\
\text { U-235 Burned, g }\end{array}$ & $\begin{array}{l}0 \\
0 \\
\end{array}$ & $\begin{array}{r}5 \\
10 \\
\end{array}$ & $\begin{array}{l}10 \\
20\end{array}$ & $\begin{array}{l}20 \\
40 \\
\end{array}$ & $\begin{array}{l}30 \\
60 \\
\end{array}$ & $\begin{array}{l}40 \\
80\end{array}$ & $\begin{array}{r}50 \\
100 \\
\end{array}$ & $\begin{array}{r}60 \\
120 \\
\end{array}$ & $\begin{array}{r}70 \\
140 \\
\end{array}$ & $\begin{array}{r}80 \\
160 \\
\end{array}$ \\
\hline U-234 & 0 & 0 & 0 & 0 & 0 & 0 & 0 & 0 & 0 & 0 \\
\hline U-235 & 200 & 190 & 180 & 160 & 140 & 120 & 100 & 80 & 60 & 40 \\
\hline U-236 & 0 & 2 & 3 & 6 & 10 & 13 & 16 & 19 & 21 & 24 \\
\hline$U-238$ & 15 & 15 & 15 & 15 & 15 & 15 & 15 & 15 & 14 & 14 \\
\hline $\mathrm{U}$ & 215 & 207 & 198 & 181 & 164 & 147 & 130 & 113 & 96 & 78 \\
\hline$N p-237$ & 0 & 0.0 & 0.0 & 0.0 & 0.1 & 0.2 & 0.3 & 0.4 & 0.6 & 0.8 \\
\hline$N p$ & 0 & 0.0 & 0.0 & 0.0 & 0.1 & 0.2 & 0.3 & 0.4 & 0.6 & 0.8 \\
\hline Pu-238 & 0 & 0.0 & 0.0 & 0.0 & 0.0 & 0.0 & 0.0 & 0.1 & 0.1 & 0.1 \\
\hline Pu-239 & 0 & 0.0 & 0.1 & 0.1 & 0.2 & 0.2 & 0.2 & 0.3 & 0.3 & 0.3 \\
\hline Pu-240 & 0 & 0.0 & 0.0 & 0.0 & 0.0 & 0.0 & 0.0 & 0.1 & 0.1 & 0.1 \\
\hline Pu-241 & 0 & 0.0 & 0.0 & 0.0 & 0.0 & 0.0 & 0.0 & 0.0 & 0.0 & 0.0 \\
\hline Pu-242 & 0 & 0.0 & 0.0 & 0.0 & 0.0 & 0.0 & 0.0 & 0.0 & 0.0 & 0.0 \\
\hline $\mathrm{Pu}$ & 0 & 0.0 & 0.1 & 0.2 & 0.2 & 0.3 & 0.3 & 0.4 & 0.5 & 0.6 \\
\hline Am-241 & 0 & 0.0 & 0.0 & 0.0 & 0.0 & 0.0 & 0.0 & 0.0 & 0.0 & 0.0 \\
\hline Am & 0 & 0.0 & 0.0 & 0.0 & 0.0 & 0.0 & 0.0 & 0.0 & 0.0 & 0.0 \\
\hline
\end{tabular}


Table 2. MTR Fuel $93 \%$ Enrichment (conti.)

\begin{tabular}{|c|c|c|c|c|c|c|c|c|c|c|}
\hline \multirow{2}{*}{$\begin{array}{l}\text { MTR Fuel } \\
\text { U-235 Bumup, \% } \\
\text { U-235 Bumed, } g\end{array}$} & \multicolumn{2}{|c|}{ 93\% Enrichment } & \multicolumn{3}{|c|}{$300 \mathrm{~g} \mathrm{U}-235$} & \multirow[b]{2}{*}{$\begin{array}{r}40 \\
120\end{array}$} & \multirow[b]{2}{*}{$\begin{array}{r}50 \\
150\end{array}$} & \multirow[b]{2}{*}{$\begin{array}{r}60 \\
180 \\
\end{array}$} & \multirow[b]{2}{*}{$\begin{array}{r}70 \\
210 \\
\end{array}$} & \multirow[b]{2}{*}{$\begin{array}{r}80 \\
240\end{array}$} \\
\hline & $\begin{array}{l}0 \\
0 \\
\end{array}$ & $\begin{array}{r}5 \\
15 \\
\end{array}$ & $\begin{array}{l}10 \\
30 \\
\end{array}$ & $\begin{array}{l}20 \\
60 \\
\end{array}$ & $\begin{array}{l}30 \\
90 \\
\end{array}$ & & & & & \\
\hline $\mathrm{U}-234$ & 0 & 0 & 0 & 0 & 0 & 0 & 0 & 0 & 0 & 0 \\
\hline U-235 & 300 & 285 & 270 & 240 & 210 & 180 & 150 & 120 & 90 & 60 \\
\hline U-236 & 0 & 3 & 5 & 10 & 15 & 19 & 24 & 28 & 33 & 37 \\
\hline U-238 & 23 & 23 & 22 & 22 & 22 & 22 & 22 & 21 & 21 & 21 \\
\hline $\mathbf{U}$ & 323 & 310 & 297 & 272 & 247 & 221 & 196 & 170 & 144 & 118 \\
\hline Np-237 & 0 & 0.0 & 0.0 & 0.1 & 0.2 & 0.4 & 0.6 & 0.8 & 1.1 & 1.5 \\
\hline $\mathrm{Np}$ & 0 & 0.0 & 0.0 & 0.1 & 0.2 & 0.4 & 0.6 & 0.8 & 1.1 & 1.5 \\
\hline Pu-238 & 0 & 0.0 & 0.0 & 0.0 & 0.0 & 0.0 & 0.1 & 0.1 & 0.2 & 0.3 \\
\hline Pu-239 & 0 & 0.1 & 0.2 & 0.3 & 0.4 & 0.4 & 0.5 & 0.5 & 0.5 & 0.5 \\
\hline Pu-240 & 0 & 0.0 & 0.0 & 0.0 & 0.0 & 0.1 & 0.1 & 0.1 & 0.1 & 0.2 \\
\hline Pu-241 & 0 & 0.0 & 0.0 & 0.0 & 0.0 & 0.0 & 0.0 & 0.1 & 0.1 & 0.1 \\
\hline Pu-242 & 0 & 0.0 & 0.0 & 0.0 & 0.0 & 0.0 & 0.0 & 0.0 & 0.0 & 0.0 \\
\hline $\mathrm{Pu}$ & 0 & 0.1 & 0.2 & 0.3 & 0.4 & 0.5 & 0.7 & 0.8 & 0.9 & 1.1 \\
\hline Am-241 & 0 & 0.0 & 0.0 & 0.0 & 0.0 & 0.0 & 0.0 & 0.0 & 0.0 & 0.0 \\
\hline Am & 0 & 0.0 & 0.0 & 0.0 & 0.0 & 0.0 & 0.0 & 0.0 & 0.0 & 0.0 \\
\hline MTR Fuel & \multicolumn{2}{|c|}{ 93\% Enrichment } & \multicolumn{3}{|c|}{$400 \mathrm{~g} \mathrm{U}-235$} & & & & & \\
\hline $\begin{array}{l}\text { U-235 Burnup, \% } \\
\text { U-235 Burned, g }\end{array}$ & $\begin{array}{l}0 \\
0\end{array}$ & $\begin{array}{r}5 \\
20 \\
\end{array}$ & $\begin{array}{l}10 \\
40 \\
\end{array}$ & $\begin{array}{l}20 \\
80 \\
\end{array}$ & $\begin{array}{r}30 \\
120 \\
\end{array}$ & $\begin{array}{r}40 \\
160 \\
\end{array}$ & $\begin{array}{r}50 \\
200 \\
\end{array}$ & $\begin{array}{r}60 \\
240 \\
\end{array}$ & $\begin{array}{r}70 \\
280 \\
\end{array}$ & $\begin{array}{r}80 \\
320 \\
\end{array}$ \\
\hline U-234 & 0 & 0 & 0 & 0 & 0 & 0 & 0 & 0 & 0 & 0 \\
\hline U-235 & 400 & 380 & 360 & 320 & 280 & 240 & 200 & 160 & 120 & 80 \\
\hline$U-236$ & 0 & 3 & 7 & 14 & 20 & 26 & 33 & 39 & 44 & 50 \\
\hline $\mathrm{U}-238$ & 30 & 30 & 30 & 30 & 29 & 29 & 29 & 28 & 28 & 27 \\
\hline U & 430 & 413 & 397 & 363 & 329 & 295 & 261 & 227 & 192 & 157 \\
\hline Np-237 & 0 & 0.0 & 0.0 & 0.2 & 0.4 & 0.6 & 0.9 & 1.3 & 1.7 & 2.2 \\
\hline $\mathrm{Np}$ & 0 & 0.0 & 0.0 & 0.2 & 0.4 & 0.6 & 0.9 & 1.3 & 1.7 & 2.2 \\
\hline Pu-238 & $\therefore 0$ & 0.0 & 0.0 & 0.0 & 0.0 & 0.1 & 0.1 & 0.2 & 0.3 & 0.5 \\
\hline Pu-239 & 0 & 0.1 & 0.2 & 0.4 & 0.6 & 0.7 & 0.7 & 0.7 & 0.7 & 0.7 \\
\hline$P u-240$ & 0 & 0.0 & 0.0 & 0.0 & 0.1 & 0.1 & 0.1 & 0.2 & 0.2 & 0.2 \\
\hline Pu-241 & 0 & 0.0 & 0.0 & 0.0 & 0.0 & 0.0 & 0.1 & 0.1 & 0.1 & 0.1 \\
\hline Pu-242 & 0 & 0.0 & 0.0 & 0.0 & 0.0 & 0.0 & 0.0 & 0.0 & 0.0 & 0.1 \\
\hline $\mathrm{Pu}$ & 0 & 0.1 & 0.3 & 0.5 & 0.7 & 0.9 & 1.1 & 1.2 & 1.4 & 1.7 \\
\hline Am-241 & 0 & 0.0 & 0.0 & 0.0 & 0.0 & 0.0 & 0.0 & 0.0 & 0.0 & 0.0 \\
\hline Am & 0 & 0.0 & 0.0 & 0.0 & 0.0 & 0.0 & 0.0 & 0.0 & 0.0 & 0.0 \\
\hline
\end{tabular}


Table 3. MTR Fuel 45\% Enrichment

\begin{tabular}{|c|c|c|c|c|c|c|c|c|c|c|}
\hline \multirow{2}{*}{$\begin{array}{l}\text { MTR Fuel } \\
\text { U-235 Bumup, \% } \\
\text { U-235 Burned, } g\end{array}$} & \multicolumn{3}{|c|}{$45 \%$ Enrichment } & \multicolumn{2}{|c|}{$200 \mathrm{~g} \mathrm{U}-235$} & \multirow[b]{2}{*}{$\begin{array}{l}40 \\
80 \\
\end{array}$} & \multirow[b]{2}{*}{$\begin{array}{r}50 \\
100 \\
\end{array}$} & \multirow[b]{2}{*}{$\begin{array}{r}60 \\
120 \\
\end{array}$} & \multirow[b]{2}{*}{$\begin{array}{r}70 \\
140 \\
\end{array}$} & \multirow[b]{2}{*}{$\begin{array}{r}80 \\
160 \\
\end{array}$} \\
\hline & $\begin{array}{l}0 \\
0\end{array}$ & $\begin{array}{r}5 \\
10\end{array}$ & $\begin{array}{l}10 \\
20\end{array}$ & $\begin{array}{l}20 \\
40\end{array}$ & $\begin{array}{l}30 \\
60\end{array}$ & & & & & \\
\hline U-234 & 0 & 0 & 0 & 0 & 0 & 0 & 0 & 0 & 0 & 0 \\
\hline U-235 & 200 & 190 & 180 & 160 & 140 & 120 & 100 & 80 & 60 & 40 \\
\hline U-236 & 0 & 2 & 3 & 6 & 10 & 13 & 16 & 19 & 21 & 24 \\
\hline U-238 & 244 & 244 & 244 & 243 & 242 & 241 & 240 & 239 & 237 & 236 \\
\hline$U$ & 444 & 436 & 427 & 409 & 391 & 374 & 356 & 337 & 319 & 300 \\
\hline Np-237 & 0 & 0.0 & 0.0 & 0.0 & 0.1 & 0.2 & 0.3 & 0.4 & 0.6 & 0.8 \\
\hline Np & 0 & 0.0 & 0.0 & 0.0 & 0.1 & 0.2 & 0.3 & 0.4 & 0.6 & 0.8 \\
\hline Pu-238 & 0 & 0.0 & 0.0 & 0.0 & 0.0 & 0.0 & 0.0 & 0.1 & 0.1 & 0.2 \\
\hline Pu-239 & 0 & 0.4 & 0.7 & 1.3 & 1.7 & 2.0 & 2.3 & 2.4 & 2.3 & 2.2 \\
\hline Pu-240 & 0 & 0.0 & 0.0 & 0.1 & 0.2 & 0.3 & 0.5 & 0.6 & 0.8 & 0.9 \\
\hline Pu-241 & 0 & 0.0 & 0.0 & 0.0 & 0.0 & 0.1 & 0.2 & 0.2 & 0.3 & 0.4 \\
\hline Pu-242 & 0 & 0.0 & 0.0 & 0.0 & 0.0 & 0.0 & 0.0 & 0.0 & 0.1 & 0.2 \\
\hline $\mathrm{Pu}$ & 0 & 0.4 & 0.7 & 1.4 & 2.0 & 2.5 & 2.9 & 3.3 & 3.6 & 3.8 \\
\hline Am-241 & 0 & 0.0 & 0.0 & 0.0 & 0.0 & 0.0 & 0.0 & 0.0 & 0.0 & 0.0 \\
\hline Am & 0 & 0.0 & 0.0 & 0.0 & 0.0 & 0.0 & 0.0 & 0.0 & 0.0 & 0.0 \\
\hline MTR Fuel & \multicolumn{3}{|c|}{ 45\% Enrichment } & \multicolumn{2}{|c|}{$300 \mathrm{~g} \mathrm{U}-235$} & & & & & \\
\hline $\begin{array}{l}\text { U-235 Bumup, \% } \\
\text { U-235 Burned, } g\end{array}$ & $\begin{array}{l}0 \\
0\end{array}$ & $\begin{array}{r}5 \\
15 \\
\end{array}$ & $\begin{array}{l}10 \\
30 \\
\end{array}$ & $\begin{array}{l}20 \\
60 \\
\end{array}$ & $\begin{array}{l}30 \\
90 \\
\end{array}$ & $\begin{array}{r}40 \\
120 \\
\end{array}$ & $\begin{array}{r}50 \\
150 \\
\end{array}$ & $\begin{array}{r}60 \\
180 \\
\end{array}$ & $\begin{array}{r}70 \\
210 \\
\end{array}$ & $\begin{array}{r}80 \\
240 \\
\end{array}$ \\
\hline U.234 & 0 & 0 & 0 & 0 & 0 & 0 & 0 & 0 & 0 & 0 \\
\hline U-235 & 300 & 285 & 270 & 240 & 210 & 180 & 150 & 120 & 90 & 60 \\
\hline U-236 & 0 & 3 & 5 & 10 & 15 & 19 & 24 & 29 & 33 & 37 \\
\hline U-238 & 367 & 366 & 365 & 364 & 362 & 361 & 359 & 357 & 355 & 352 \\
\hline U & 667 & 654 & 640 & 614 & 587 & 560 & 533 & 505 & 477 & 449 \\
\hline Np-237 & 0 & 0.0 & 0.0 & 0.1 & 0.2 & 0.4 & 0.6 & 0.9 & 1.2 & 1.5 \\
\hline$N p$ & 0 & 0.0 & 0.0 & 0.1 & 0.2 & 0.4 & 0.6 & 0.9 & 1.2 & 1.5 \\
\hline Pu-238 & $\therefore 0$ & 0.0 & 0.0 & 0.0 & 0.0 & 0.0 & 0.1 & 0.1 & 0.2 & 0.3 \\
\hline Pu-239 & 0 & 0.6 & 1.2 & 2.2 & 2.9 & 3.4 & 3.8 & 3.9 & 3.8 & 3.6 \\
\hline Pu-240 & 0 & 0.0 & 0.0 & 0.2 & 0.3 & 0.6 & 0.8 & 1.0 & 1.2 & 1.4 \\
\hline Pu-241 & 0 & 0.0 & 0.0 & 0.0 & 0.1 & 0.2 & 0.3 & 0.5 & 0.6 & 0.7 \\
\hline Pu-242 & 0 & 0.0 & 0.0 & 0.0 & 0.0 & 0.0 & 0.0 & 0.1 & 0.2 & 0.3 \\
\hline $\mathrm{Pu}$ & 0 & 0.6 & 1.3 & 2.4 & 3.4 & 4.2 & 5.0 & 5.6 & 6.0 & 6.3 \\
\hline$A m-241$ & 0 & 0.0 & 0.0 & 0.0 & 0.0 & 0.0 & 0.0 & 0.0 & 0.0 & 0.0 \\
\hline Am & 0 & 0.0 & 0.0 & 0.0 & 0.0 & 0.0 & 0.0 & 0.0 & 0.0 & 0.0 \\
\hline
\end{tabular}


Table 3. MTR Fuel 45\% Enrichment (conti.)

\begin{tabular}{|c|c|c|c|c|c|c|c|c|c|c|}
\hline \multirow{2}{*}{$\begin{array}{l}\text { MTR Fuel } \\
\text { U-235 Bumup, \% } \\
\text { U-235 Bumed, g }\end{array}$} & \multicolumn{3}{|c|}{$45 \%$ Enrichment } & \multicolumn{2}{|c|}{$400 \mathrm{~g} \mathrm{U}-235$} & \multirow[b]{2}{*}{$\begin{array}{r}40 \\
160\end{array}$} & \multirow[b]{2}{*}{$\begin{array}{r}50 \\
200 \\
\end{array}$} & \multirow[b]{2}{*}{$\begin{array}{r}60 \\
240 \\
\end{array}$} & \multirow[b]{2}{*}{$\begin{array}{r}70 \\
280 \\
\end{array}$} & \multirow[b]{2}{*}{$\begin{array}{r}80 \\
320 \\
\end{array}$} \\
\hline & $\begin{array}{l}0 \\
0\end{array}$ & $\begin{array}{r}5 \\
20 \\
\end{array}$ & $\begin{array}{l}10 \\
40\end{array}$ & $\begin{array}{l}20 \\
80\end{array}$ & $\begin{array}{r}30 \\
120\end{array}$ & & & & & \\
\hline U-234 & 0 & 0 & 0 & 0 & 0 & 0 & 0 & 0 & 0 & 0 \\
\hline U-235 & 400 & 380 & 360 & 320 & 280 & 240 & 200 & 160 & 120 & 80 \\
\hline U-236 & 0 & 3 & 7 & 14 & 20 & 27 & 33 & 39 & 45 & 50 \\
\hline U-238 & 489 & 488 & 487 & 485 & 482 & 480 & 477 & 474 & 471 & 467 \\
\hline$U$ & 889 & 871 & 854 & 818 & 782 & 746 & 710 & 673 & 636 & 597 \\
\hline Np-237 & 0 & 0.0 & 0.0 & 0.2 & 0.4 & 0.6 & 1.0 & 1.4 & 1.8 & 2.4 \\
\hline $\mathrm{Np}$ & 0 & 0.0 & 0.0 & 0.2 & 0.4 & 0.6 & 1.0 & 1.4 & 1.8 & 2.4 \\
\hline Pu-238 & 0 & 0.0 & 0.0 & 0.0 & 0.0 & 0.1 & 0.1 & 0.2 & 0.3 & 0.5 \\
\hline Pu-239 & 0 & 0.9 & 1.8 & 3.2 & 4.2 & 4.9 & 5.4 & 5.5 & 5.4 & 5.0 \\
\hline Pu-240 & 0 & 0.0 & 0.1 & 0.3 & 0.5 & 0.8 & 1.1 & 1.4 & 1.6 & 1.9 \\
\hline $\mathrm{Pu}-241$ & 0 & 0.0 & 0.0 & 0.1 & 0.2 & 0.3 & 0.6 & 0.8 & 1.0 & 1.1 \\
\hline Pu-242 & 0 & 0.0 & 0.0 & 0.0 & 0.0 & 0.0 & 0.1 & 0.2 & 0.3 & 0.5 \\
\hline $\mathrm{Pu}$ & 0 & 0.9 & 1.9 & 3.5 & 4.9 & 6.2 & 7.2 & 8.1 & 8.7 & 9.1 \\
\hline Am-241 & 0 & 0.0 & 0.0 & 0.0 & 0.0 & 0.0 & 0.0 & 0.0 & 0.0 & 0.0 \\
\hline Am & 0 & 0.0 & 0.0 & 0.0 & 0.0 & 0.0 & 0.0 & 0.0 & 0.0 & 0.0 \\
\hline
\end{tabular}


Table 4. MTR Fuel $19.75 \%$ Enrichment

\begin{tabular}{|c|c|c|c|c|c|c|c|c|c|c|}
\hline MTR Fuel & $19.75 \%$ & ment & & 100 & & & & & & \\
\hline $\begin{array}{l}\text { U-235 Burnup, \% } \\
\text { U-235 Burned, g }\end{array}$ & $\begin{array}{l}0 \\
0\end{array}$ & $\begin{array}{l}5 \\
5\end{array}$ & $\begin{array}{l}10 \\
10 \\
\end{array}$ & $\begin{array}{l}20 \\
20\end{array}$ & $\begin{array}{l}30 \\
30\end{array}$ & $\begin{array}{l}40 \\
40\end{array}$ & $\begin{array}{l}50 \\
50\end{array}$ & $\begin{array}{l}60 \\
60\end{array}$ & $\begin{array}{l}70 \\
70\end{array}$ & $\begin{array}{l}80 \\
80 \\
\end{array}$ \\
\hline $\mathrm{U}-234$ & 0 & 0 & 0 & 0 & 0 & 0 & 0 & 0 & 0 & 0 \\
\hline U-235 & 100. & 95 & 90 & 80 & 70 & 60 & 50 & 40 & 30 & 20 \\
\hline U-236 & 0 & 1 & 2 & 3 & 5 & 6 & 8 & 9 & 11 & 12 \\
\hline U-238 & 406 & 406 & 406 & 405 & 404 & 403 & 402 & 401 & 399 & 398 \\
\hline $\mathbf{U}$ & 506 & 502 & 497 & 488 & 479 & 469 & 460 & 450 & 440 & 429 \\
\hline Np-237 & 0 & 0.0 & 0.0 & 0.0 & 0.0 & 0.1 & 0.1 & 0.2 & 0.2 & 0.3 \\
\hline Np & 0 & 0.0 & 0.0 & 0.0 & 0.0 & 0.1 & 0.1 & 0.2 & 0.2 & 0.3 \\
\hline Pu-238 & 0 & 0.0 & 0.0 & 0.0 & 0.0 & 0.0 & 0.0 & 0.0 & 0.0 & 0.1 \\
\hline Pu-239 & 0 & 0.3 & 0.7 & 1.2 & 1.7 & 2.0 & 2.3 & 2.5 & 2.5 & 2.5 \\
\hline Pu-240 & 0 & 0.0 & 0.0 & 0.1 & 0.2 & 0.3 & 0.4 & 0.6 & 0.8 & 1.0 \\
\hline Pu-241 & 0 & $0.0^{\circ}$ & 0.0 & 0.0 & 0.0 & 0.1 & 0.1 & 0.2 & 0.2 & 0.3 \\
\hline Pu-242 & 0 & 0.0 & 0.0 & 0.0 & 0.0 & 0.0 & 0.0 & 0.0 & 0.1 & 0.1 \\
\hline $\mathrm{Pu}$ & 0 & 0.3 & 0.7 & 1.3 & 1.9 & 2.4 & 2.9 & 3.3 & 3.7 & 4.0 \\
\hline Am-241 & 0 & 0.0 & 0.0 & 0.0 & 0.0 & 0.0 & 0.0 & 0.0 & 0.0 & 0.0 \\
\hline $\mathrm{Am}$ & 0 & 0.0 & 0.0 & 0.0 & 0.0 & 0.0 & 0.0 & 0.0 & 0.0 & 0.0 \\
\hline
\end{tabular}

MTR Fuel

$19.75 \%$ Enrichment $200 \mathrm{~g} \mathrm{U}-235$

\begin{tabular}{|c|c|c|c|c|c|c|c|c|c|c|}
\hline $\begin{array}{l}\text { U-235 Burnup, \% } \\
\text { U-235 Burned, } g \\
\end{array}$ & $\begin{array}{l}0 \\
0 \\
\end{array}$ & $\begin{array}{r}5 \\
10 \\
\end{array}$ & $\begin{array}{l}10 \\
20 \\
\end{array}$ & $\begin{array}{l}20 \\
40 \\
\end{array}$ & $\begin{array}{l}30 \\
60 \\
\end{array}$ & $\begin{array}{l}40 \\
80 \\
\end{array}$ & $\begin{array}{r}50 \\
100 \\
\end{array}$ & $\begin{array}{r}60 \\
120 \\
\end{array}$ & $\begin{array}{r}70 \\
140 \\
\end{array}$ & $\begin{array}{r}80 \\
160 \\
\end{array}$ \\
\hline U-234 & 0 & 0 & 0 & 0 & 0 & 0 & 0 & 0 & 0 & 0 \\
\hline U-235 & 200 & 190 & 180 & 160 & 140 & 120 & 100 & 80 & 60 & 40 \\
\hline U-236 & 0 & 2 & 3 & 6 & 10 & 13 & 16 & 19 & 22 & 24 \\
\hline U-238 & 813 & 812 & 811 & 809 & 807 & 805 & 802 & 800 & 796 & 792 \\
\hline U & 1013 & 1003 & 994 & 975 & 957 & 937 & 918 & 898 & 878 & 856 \\
\hline Np-237 & 0 & 0.0 & 0.0 & 0.0 & 0.1 & 0.2 & 0.3 & 0.5 & 0.6 & 0.9 \\
\hline Np & 0 & 0.0 & 0.0 & 0.0 & 0.1 & 0.2 & 0.3 & 0.5 & 0.6 & 0.9 \\
\hline Pu-238 & $\therefore 0$ & 0.0 & 0.0 & 0.0 & 0.0 & 0.0 & 0.0 & 0.1 & 0.1 & 0.2 \\
\hline Pu-239 & 0 & 0.8 & 1.5 & 2.8 & 3.8 & 4.6 & 5.1 & 5.4 & 5.5 & 5.3 \\
\hline Pu-240 & 0 & 0.0 & 0.1 & 0.2 & 0.4 & 0.7 & 1.0 & 1.4 & 1.7 & 2.1 \\
\hline Pu-241 & 0 & 0.0 & 0.0 & 0.0 & 0.1 & 0.2 & 0.3 & 0.5 & 0.7 & 0.9 \\
\hline Pu-242 & 0 & 0.0 & 0.0 & 0.0 & 0.0 & 0.0 & 0.0 & 0.1 & 0.2 & 0.4 \\
\hline $\mathrm{Pu}$ & 0 & 0.8 & 1.6 & 3.1 & 4.4 & 5.5 & 6.6 & 7.5 & 8.2 & 8.8 \\
\hline Am-241 & 0 & 0.0 & 0.0 & 0.0 & 0.0 & 0.0 & 0.0 & 0.0 & 0.0 & 0.0 \\
\hline Am & 0 & 0.0 & 0.0 & 0.0 & 0.0 & 0.0 & 0.0 & 0.0 & 0.0 & 0.0 \\
\hline
\end{tabular}


Table 4. MTR Fuel $19.75 \%$ Enrichment (conti.)

\begin{tabular}{|c|c|c|c|c|c|c|c|c|c|c|}
\hline \multirow{2}{*}{$\begin{array}{l}\text { MTR Fue! } \\
\text { U-235 Burnup, \% } \\
\text { U-235 Burned, g }\end{array}$} & \multicolumn{3}{|c|}{$19.75 \%$ Enrichment } & \multicolumn{2}{|c|}{$300 \mathrm{~g} \mathrm{U}-235$} & \multirow[b]{2}{*}{$\begin{array}{r}40 \\
120 \\
\end{array}$} & \multirow[b]{2}{*}{$\begin{array}{r}50 \\
150 \\
\end{array}$} & \multirow[b]{2}{*}{$\begin{array}{r}60 \\
180 \\
\end{array}$} & \multirow[b]{2}{*}{$\begin{array}{r}70 \\
210 \\
\end{array}$} & \multirow[b]{2}{*}{$\begin{array}{r}80 \\
240 \\
\end{array}$} \\
\hline & $\begin{array}{l}0 \\
0\end{array}$ & $\begin{array}{r}5 \\
15\end{array}$ & $\begin{array}{l}10 \\
30\end{array}$ & $\begin{array}{l}20 \\
60\end{array}$ & $\begin{array}{l}30 \\
90\end{array}$ & & & & & \\
\hline U-234 & 0 & 0 & 0 & 0 & 0 & 0 & 0 & 0 & 0 & 0 \\
\hline U-235 & 300 & 285 & 270 & 240 & 210 & 180 & 150 & 120 & 90 & 60 \\
\hline U-236 & 0 & 3 & 5 & 10 & 15 & 20 & 24 & 29 & 33 & 37 \\
\hline U-238 & 1219 & 1218 & 1216 & 1213 & 1209 & 1205 & 1201 & 1197 & 1191 & 1184 \\
\hline$U$ & 1519 & 1505 & 1491 & 1463 & 1434 & 1405 & 1375 & 1345 & 1314 & 1281 \\
\hline Np-237 & 0 & 0.0 & 0.0 & 0.1 & 0.2 & 0.4 & 0.6 & 0.9 & 1.2 & 1.6 \\
\hline $\mathrm{Np}$ & 0 & 0.0 & 0.0 & 0.1 & 0.2 & 0.4 & 0.6 & 0.9 & 1.2 & 1.6 \\
\hline Pu-238 & 0 & 0.0 & 0.0 & 0.0 & 0.0 & 0.0 & 0.1 & 0.1 & 0.2 & 0.3 \\
\hline Pu-239 & 0 & 1.3 & 2.6 & 4.7 & 6.3 & 7.5 & 8.3 & 8.7 & 8.7 & 8.4 \\
\hline Pu-240 & 0 & 0.0 & 0.1 & 0.4 & 0.7 & 1.2 & 1.7 & 2.2 & 2.7 & 3.2 \\
\hline Pu-241 & 0 & 0.0 & 0.0 & 0.1 & 0.2 & 0.4 & 0.7 & 1.0 & 1.4 & 1.6 \\
\hline Pu-242 & 0 & 0.0 & 0.0 & 0.0 & 0.0 & 0.0 & 0.1 & 0.2 & 0.4 & 0.7 \\
\hline $\mathrm{Pu}$ & 0 & 1.4 & 2.7 & 5.1 & 7.3 & 9.2 & 10.9 & 12.3 & 13.4 & 14.3 \\
\hline Am-241 & 0 & 0.0 & 0.0 & 0.0 & 0.0 & 0.0 & 0.0 & 0.0 & 0.0 & 0.1 \\
\hline $\mathrm{Am}$ & 0 & 0.0 & 0.0 & 0.0 & 0.0 & 0.0 & 0.0 & 0.0 & 0.0 & 0.1 \\
\hline MTR Fuel & $19.75 \%$ & hment & & 400 & 35 & & & & & \\
\hline $\begin{array}{l}\text { U-235 Burnup, \% } \\
\text { U-235 Burned, g }\end{array}$ & $\begin{array}{l}0 \\
0 \\
\end{array}$ & $\begin{array}{r}5 \\
20 \\
\end{array}$ & $\begin{array}{l}10 \\
40 \\
\end{array}$ & $\begin{array}{l}20 \\
80 \\
\end{array}$ & $\begin{array}{r}30 \\
120 \\
\end{array}$ & $\begin{array}{r}40 \\
160 \\
\end{array}$ & $\begin{array}{r}50 \\
200 \\
\end{array}$ & $\begin{array}{r}60 \\
240 \\
\end{array}$ & $\begin{array}{r}70 \\
280 \\
\end{array}$ & $\begin{array}{r}80 \\
320 \\
\end{array}$ \\
\hline U-234 & 0 & 0 & 0 & $\overline{0}$ & 0 & 0 & 0 & 0 & 0 & 0 \\
\hline U-235 & 400 & 380 & 360 & 320 & 280 & 240 & 200 & 160 & 120 & 80 \\
\hline U-236 & 0 & 4 & 7 & 14 & 20 & 27 & 33 & 39 & 45 & 50 \\
\hline U-238 & 1625 & 1623 & 1621 & 1616 & 1611 & 1605 & 1599 & 1592 & 1584 & 1574 \\
\hline$U$ & 2025 & 2007 & 1988 & 1950 & 1911 & 1872 & 1832 & 1791 & 1749 & 1704 \\
\hline Np-237 & 0 & 0.0 & 0.0 & 0.2 & 0.4 & 0.7 & 1.0 & 1.4 & 1.9 & 2.5 \\
\hline$N p$ & 0 & 0.0 & 0.0 & 0.2 & 0.4 & 0.7 & 1.0 & 1.4 & 1.9 & 2.5 \\
\hline Pu-238 & $\therefore 0$ & 0.0 & 0.0 & 0.0 & 0.0 & 0.1 & 0.1 & 0.2 & 0.4 & 0.6 \\
\hline Pu-239 & 0 & 2.0 & 3.8 & 6.8 & 9.1 & 10.8 & 11.8 & 12.4 & 12.3 & 11.7 \\
\hline Pu-240 & 0 & 0.0 & 0.2 & 0.6 & 1.1 & 1.7 & 2.4 & 3.1 & 3.7 & 4.3 \\
\hline Pu-241 & 0 & 0.0 & 0.0 & 0.1 & 0.4 & 0.7 & 1.2 & 1.7 & 2.2 & 2.6 \\
\hline $\mathrm{Pu}-242$ & 0 & 0.0 & 0.0 & 0.0 & 0.0 & 0.1 & 0.2 & 0.4 & 0.7 & 1.2 \\
\hline $\mathrm{Pu}$ & 0 & 2.0 & 3.9 & 7.5 & 10.6 & 13.4 & 15.8 & 17.7 & 19.3 & 20.4 \\
\hline Am-241 & 0 & 0.0 & 0.0 & 0.0 & 0.0 & 0.0 & 0.0 & 0.0 & 0.1 & 0.1 \\
\hline Am & 0 & 0.0 & 0.0 & 0.0 & 0.0 & 0.0 & 0.0 & 0.0 & 0.1 & 0.1 \\
\hline
\end{tabular}


Table 4. MTR Fuel $19.75 \%$ Enrichment (conti.)

\begin{tabular}{|c|c|c|c|c|c|c|c|c|c|c|}
\hline \multirow{2}{*}{$\begin{array}{l}\text { MTR Fuel } \\
\text { U-235 Bumup, \% } \\
\text { U-235 Bumed, g }\end{array}$} & \multicolumn{2}{|c|}{$19.75 \%$ Enrichment } & \multicolumn{3}{|c|}{$500 \mathrm{~g} \mathrm{U}-235$} & \multirow{2}{*}{$\begin{array}{r}40 \\
200\end{array}$} & \multirow[b]{2}{*}{$\begin{array}{r}50 \\
250\end{array}$} & \multirow[b]{2}{*}{$\begin{array}{r}60 \\
300\end{array}$} & \multirow[b]{2}{*}{$\begin{array}{r}70 \\
350\end{array}$} & \multirow[b]{2}{*}{$\begin{array}{r}80 \\
400\end{array}$} \\
\hline & $\begin{array}{l}0 \\
0\end{array}$ & $\begin{array}{r}5 \\
25\end{array}$ & $\begin{array}{l}10 \\
50\end{array}$ & $\begin{array}{r}20 \\
100\end{array}$ & $\begin{array}{r}30 \\
150\end{array}$ & & & & & \\
\hline U-234 & 0 & 0 & 0 & 0 & 0 & 0 & 0 & 0 & 0 & 0 \\
\hline U-235 & 500 & 475 & 450 & 400 & 350 & 300 & 250 & 200 & 150 & 100 \\
\hline U-236 & 0 & 4 & 9 & 18 & 26 & 34 & 42 & 50 & 57 & 64 \\
\hline$U-238$ & 2032 & 2029 & 2026 & 2019 & 2012 & 2004 & 1996 & 1987 & 1976 & 1962 \\
\hline U & 2532 & 2508 & 2484 & 2437 & 2388 & 2338 & 2288 & 2236 & 2183 & 2126 \\
\hline Np-237 & 0 & 0.0 & 0.1 & 0.3 & 0.6 & 1.0 & 1.5 & 2.1 & 2.8 & 3.6 \\
\hline $\mathrm{Np}$ & 0 & 0.0 & 0.1 & 0.3 & 0.6 & 1.0 & 1.5 & 2.1 & 2.8 & 3.6 \\
\hline Pu-238 & 0 & 0.0 & 0.0 & 0.0 & 0.0 & 0.1 & 0.2 & 0.4 & 0.6 & 0.9 \\
\hline Pu-239 & 0 & 2.6 & 5.0 & 9.0 & 12.1 & 14.3 & 15.6 & 16.2 & 16.1 & 15.3 \\
\hline Pu-240 & 0 & 0.1 & 0.2 & 0.8 & 1.5 & 2.3 & 3.2 & 4.0 & 4.7 & 5.4 \\
\hline Pu-241 & 0 & 0.0 & 0.0 & 0.2 & 0.6 & 1.1 & 1.8 & 2.5 & 3.2 & 3.6 \\
\hline Pu-242 & 0 & 0.0 & 0.0 & 0.0 & 0.0 & 0.1 & 0.3 & 0.6 & 1.0 & 1.7 \\
\hline $\mathrm{Pu}$ & 0 & 2.7 & 5.3 & 10.0 & 14.2 & 17.9 & 21.1 & 23.7 & 25.7 & 27.0 \\
\hline Am-241 & 0 & 0.0 & 0.0 & 0.0 & 0.0 & 0.0 & 0.0 & 0.1 & 0.1 & 0.1 \\
\hline $\mathrm{Am}$ & 0 & 0.0 & 0.0 & 0.0 & 0.0 & 0.0 & 0.0 & 0.1 & 0.1 & 0.1 \\
\hline
\end{tabular}


Table 5. TRIGA Fuel Single-Rod Model

\begin{tabular}{|c|c|c|c|c|c|c|c|c|c|c|c|c|c|c|c|c|c|}
\hline \multirow{2}{*}{$\begin{array}{l}\text { TRIGA Fuel } \\
\text { U-235 Burnup, \% } \\
\text { U-235 Burned, g }\end{array}$} & \multicolumn{4}{|c|}{$8.5 w t \%$ U, $70 \%$ Enrichment } & \multicolumn{3}{|c|}{$133 \mathrm{~g} \mathrm{U}-235$} & \multirow[b]{2}{*}{$\begin{array}{l}35 \\
47\end{array}$} & \multirow{2}{*}{$\begin{array}{l}\text { TRIGA Fuel } \\
\text { U-235 Bumup, \% } \\
\text { U-235 Bumed, g }\end{array}$} & \multicolumn{4}{|c|}{$20 w t \%$ U, 20\% Enrichment } & \multicolumn{3}{|c|}{$98 \mathrm{~g} \mathrm{U}-235$} & \multirow[b]{2}{*}{$\begin{array}{l}35 \\
34 \\
\end{array}$} \\
\hline & $\begin{array}{l}0 \\
0\end{array}$ & $\begin{array}{l}5 \\
7 \\
\end{array}$ & $\begin{array}{l}10 \\
13\end{array}$ & $\begin{array}{l}15 \\
20 \\
\end{array}$ & $\begin{array}{l}20 \\
27 \\
\end{array}$ & $\begin{array}{l}25 \\
33 \\
\end{array}$ & $\begin{array}{l}30 \\
40 \\
\end{array}$ & & & $\begin{array}{l}0 \\
0\end{array}$ & $\begin{array}{l}5 \\
5\end{array}$ & $\begin{array}{l}10 \\
10\end{array}$ & $\begin{array}{l}15 \\
15 \\
\end{array}$ & $\begin{array}{l}20 \\
20 \\
\end{array}$ & $\begin{array}{l}25 \\
25\end{array}$ & $\begin{array}{l}30 \\
29\end{array}$ & \\
\hline U-234 & 0 & 0 & 0 & 0 & 0 & 0 & 0 & 0 & $\overline{U-234}$ & 0 & 0 & 0 & 0 & 0 & 0 & 0 & 0 \\
\hline U-235 & 133 & 126 & 120 & 113 & 106 & 100 & 93 & 87 & U-235 & 98 & 93 & 88 & 83 & 78 & 74 & 69 & 64 \\
\hline U-236 & 0 & 1 & 3 & 4 & 5 & 6 & 7 & 8 & U-236 & 0 & 1 & 2 & 3 & 4 & 4 & 5 & 6 \\
\hline U-238 & 57 & 57 & 56 & 56 & 56 & 56 & 55 & 55 & U-238 & 392 & 391 & 391 & 390 & 389 & 388 & 388 & 387 \\
\hline$U$ & 190 & 184 & 179 & 173 & 167 & 162 & 156 & 150 & $\mathbf{U}$ & 490 & 485 & 481 & 476 & 471 & 466 & 461 & 457 \\
\hline $\mathrm{Np}-237$ & 0 & 0.0 & 0.0 & 0.1 & 0.1 & 0.1 & 0.2 & 0.3 & Np-237 & 0 & 0.0 & 0.0 & 0.0 & 0.1 & 0.1 & 0.1 & 0.2 \\
\hline $\mathrm{Np}$ & 0 & 0.0 & 0.0 & 0.1 & 0.1 & 0.1 & 0.2 & 0.3 & Np & 0 & 0.0 & 0.0 & 0.0 & 0.1 & 0.1 & 0.1 & 0.2 \\
\hline Pu-238 & 0 & 0.0 & 0.0 & 0.0 & 0.0 & 0.0 & 0.0 & 0.0 & Pu-238 & 0 & 0.0 & 0.0 & 0.0 & 0.0 & 0.0 & 0.0 & 0.0 \\
\hline Pu-239 & 0 & 0.3 & 0.5 & 0.7 & 0.8 & 0.9 & 1.0 & 1.1 & Pu-239 & 0 & 0.6 & 1.1 & 1.6 & 2.0 & 2.4 & 2.7 & 2.9 \\
\hline Pu-240 & 0 & 0.0 & 0.0 & 0.0 & 0.1 & 0.1 & 0.1 & 0.2 & Pu-240 & 0 & 0.0 & 0.1 & 0.1 & 0.2 & 0.3 & 0.3 & 0.4 \\
\hline Pu-241 & 0 & 0.0 & 0.0 & 0.0 & 0.0 & 0.0 & 0.1 & 0.1 & Pu-241 & 0 & 0.0 & 0.0 & 0.0 & 0.0 & 0.1 & 0.1 & 0.2 \\
\hline$P u-242$ & 0 & 0.0 & 0.0 & 0.0 & 0.0 & 0.0 & 0.0 & 0.0 & Pu-242 & 0 & 0.0 & 0.0 & 0.0 & 0.0 & 0.0 & 0.0 & 0.0 \\
\hline $\mathrm{Pu}$ & 0 & 0.3 & 0.5 & 0.7 & 0.9 & 1.1 & 1.2 & 1.4 & $\mathrm{Pu}$ & 0 & 0.6 & 1.2 & 1.7 & 2.3 & 2.7 & 3.2 & 3.6 \\
\hline Am-241 & 0 & 0.0 & 0.0 & 0.0 & 0.0 & 0.0 & 0.0 & 0.0 & Am-241 & 0 & 0.0 & 0.0 & 0.0 & 0.0 & 0.0 & 0.0 & 0.0 \\
\hline$A m$ & 0 & 0.0 & 0.0 & 0.0 & 0.0 & 0.0 & 0.0 & 0.0 & Am & 0 & 0.0 & 0.0 & 0.0 & 0.0 & 0.0 & 0.0 & 0.0 \\
\hline TRIGA Fuel & $12 w t \%$ & $20 \%$ & arichm & & 54 & U-235 & & & TRIGA Fuel & $8.5 w t \%$ & $20 \%$ & nrichn & & 38 & U-235 & & \\
\hline $\begin{array}{l}\text { U-235 Burnup, \% } \\
\text { U-235 Burned, g }\end{array}$ & $\begin{array}{l}0 \\
0\end{array}$ & $\begin{array}{l}5 \\
3\end{array}$ & $\begin{array}{r}10 \\
5 \\
\end{array}$ & $\begin{array}{r}15 \\
8\end{array}$ & $\begin{array}{l}20 \\
11\end{array}$ & $\begin{array}{l}25 \\
14 \\
\end{array}$ & $\begin{array}{l}30 \\
16\end{array}$ & $\begin{array}{l}35 \\
19 \\
\end{array}$ & $\begin{array}{l}\text { U-235 Bumup, \% } \\
\text { U-235 Burned, g }\end{array}$ & $\begin{array}{l}0 \\
0\end{array}$ & $\begin{array}{l}5 \\
2\end{array}$ & $\begin{array}{r}10 \\
4 \\
\end{array}$ & $\begin{array}{r}15 \\
6 \\
\end{array}$ & $\begin{array}{r}20 \\
8 \\
\end{array}$ & $\begin{array}{l}25 \\
10 \\
\end{array}$ & $\begin{array}{l}30 \\
11 \\
\end{array}$ & $\begin{array}{l}35 \\
13 \\
\end{array}$ \\
\hline U-234 & 0 & 0 & 0 & 0 & 0 & 0 & 0 & $\overline{0}$ & U-234 & 0 & 0 & 0 & 0 & 0 & 0 & $\overline{0}$ & 0 \\
\hline U-235 & 54 & 51 & 49 & 46 & 43 & 41 & 38 & 35 & U-235 & 38 & 36 & 34 & 32 & 30 & 29 & 27 & 25 \\
\hline U-236 & 0 & 0 & 1 & 1 & 2 & 2 & 3 & 3 & U-236 & 0 & 0 & 1 & 1 & 1 & 2 & 2 & 2 \\
\hline U-238 & 216 & 216 & 215 & 215 & 215 & 215 & 214 & 214 & U-238 & 152 & 152 & 152 & 151 & 151 & 151 & 151 & 151 \\
\hline U & 270 & 268 & 265 & 262 & 260 & 257 & 255 & 252 & $\mathbf{U}$ & 190 & 188 & 186 & 185 & 183 & 181 & 179 & 177 \\
\hline Np-237 & 0 & 0.0 & 0.0 & 0.0 & 0.0 & 0.0 & 0.0 & 0.1 & $\mathrm{~Np}-237$ & 0 & 0.0 & 0.0 & 0.0 & 0.0 & 0.0 & 0.0 & 0.0 \\
\hline $\mathrm{Np}$ & 0 & 0.0 & 0.0 & 0.0 & 0.0 & 0.0 & 0.0 & 0.1 & $\mathrm{~Np}$ & 0 & 0.0 & 0.0 & 0.0 & 0.0 & 0.0 & 0.0 & 0.0 \\
\hline Pu-238 & 0 & 0.0 & 0.0 & 0.0 & 0.0 & 0.0 & 0.0 & 0.0 & Pu-238 & 0 & 0.0 & 0.0 & 0.0 & 0.0 & 0.0 & 0.0 & 0.0 \\
\hline Pu-239 & 0 & 0.3 & 0.0 & 0.7 & 0.9 & 1.1 & 1.2 & 1.3 & Pu-239 & 0 & 0.2 & 0.3 & 0.5 & 0.6 & 0.7 & 0.8 & 0.9 \\
\hline Pu-240 & 0 & 0.0 & 0.0 & 0.0 & 0.1 & 0.1 & 0.1 & 0.2 & Pu-240 & 0 & 0.0 & 0.0 & 0.0 & 0.0 & 0.1 & 0.1 & 0.1 \\
\hline $\mathrm{Pu}-241$ & 0 & 0.0 & 0.0 & 0.0 & 0.0 & 0.0 & 0.0 & 0.1 & Pu-241 & 0 & 0.0 & 0.0 & 0.0 & 0.0 & 0.0 & 0.0 & 0.0 \\
\hline $\mathrm{Pu}-242$ & 0 & 0.0 & 0.0 & 0.0 & 0.0 & 0.0 & 0.0 & 0.0 & Pu-242 & 0 & 0.0 & 0.0 & 0.0 & 0.0 & 0.0 & 0.0 & 0.0 \\
\hline $\mathrm{Pu}$ & 0 & 0.3 & 0.5 & 0.8 & 1.0 & 1.2 & 1.4 & 1.6 & $\mathrm{Pu}$ & 0 & 0.2 & 0.3 & 0.5 & 0.6 & 0.8 & 0.9 & 1.0 \\
\hline Am-241 & 0 & 0.0 & 0.0 & 0.0 & 0.0 & 0.0 & 0.0 & 0.0 & Am-241 & 0 & 0.0 & 0.0 & 0.0 & 0.0 & 0.0 & 0.0 & 0.0 \\
\hline $\mathrm{Am}$ & 0 & 0.0 & 0.0 & 0.0 & 0.0 & 0.0 & 0.0 & 0.0 & $\mathrm{Am}$ & 0 & 0.0 & 0.0 & 0.0 & 0.0 & 0.0 & 0.0 & 0.0 \\
\hline
\end{tabular}


Table 6. TRIGA Fuel 25-Rod Cluster Model

\begin{tabular}{|c|c|c|c|c|c|c|c|c|c|c|c|c|c|c|c|}
\hline TRIGA Fuel & $10 w t \% U$ & $3.1 \% E$ & chment & & 41.4 & $1-235$ & & TRIGA Fuel & $45 w t \%$ U & $9.7 \% \mathrm{E}$ & richment & & 53.6 & U-235 & \\
\hline $\begin{array}{l}\text { U-235 Bumup, \% } \\
\text { U-235 Burned, g }\end{array}$ & $\begin{array}{r}0 \\
0.0\end{array}$ & $\begin{array}{r}10 \\
4.1\end{array}$ & $\begin{array}{r}20 \\
8.3\end{array}$ & $\begin{array}{r}30 \\
12.4\end{array}$ & $\begin{array}{r}40 \\
16.6\end{array}$ & $\begin{array}{r}50 \\
20.7\end{array}$ & $\begin{array}{r}60 \\
24.8 \\
\end{array}$ & $\begin{array}{l}\text { U-235 Bumup, \% } \\
\text { U-235 Bumed, g }\end{array}$ & $\begin{array}{r}0 \\
0.0\end{array}$ & $\begin{array}{r}10 \\
5.4\end{array}$ & $\begin{array}{r}20 \\
10.7\end{array}$ & $\begin{array}{r}30 \\
16.1\end{array}$ & $\begin{array}{r}40 \\
21.4\end{array}$ & $\begin{array}{r}50 \\
26.8\end{array}$ & $\begin{array}{r}60 \\
32.2\end{array}$ \\
\hline U-234 & 0.4 & 0.4 & 0.4 & 0.4 & 0.4 & 0.3 & 0.3 & U-234 & 0.4 & 0.4 & 0.4 & 0.3 & 0.3 & 0.3 & $\overline{0.3}$ \\
\hline U-235 & 41.4 & 37.2 & 33.1 & 29.0 & 24.8 & 20.7 & 16.6 & U-235 & 53.6 & 48.3 & 42.9 & 37.5 & 32.2 & 26.8 & 21.4 \\
\hline U-236 & 0.2 & 1.0 & 1.7 & 2.4 & 3.1 & 3.8 & 4.4 & U-236 & 0.7 & 1.7 & 2.7 & 3.7 & 4.6 & 5.5 & 6.4 \\
\hline U-238 & 2.4 & 2.4 & 2.4 & 2.3 & 2.3 & 2.2 & 2.2 & U-238 & 217.4 & 216.5 & 215.6 & 214.6 & 213.5 & 212.3 & 210.9 \\
\hline $\mathrm{U}$ & 44.5 & 41.0 & 37.6 & 34.1 & 30.6 & 27.1 & 23.5 & $\mathbf{U}$ & 272.1 & 266.9 & 261.6 & 256.1 & 250.6 & 244.9 & 239.0 \\
\hline $\mathrm{Np}-237$ & 0 & 0.0 & 0.0 & 0.1 & 0.1 & 0.2 & 0.2 & Np-237 & 0 & 0.0 & 0.1 & 0.1 & 0.2 & 0.3 & 0.4 \\
\hline Np & 0 & 0.0 & 0.0 & 0.1 & 0.1 & 0.2 & 0.2 & $\mathrm{~Np}$ & 0 & 0.0 & 0.1 & 0.1 & 0.2 & 0.3 & 0.4 \\
\hline Pu-238 & 0 & 0.0 & 0.0 & 0.0 & 0.0 & 0.0 & 0.0 & Pu-238 & 0 & 0.0 & 0.0 & 0.0 & 0.0 & 0.1 & 0.1 \\
\hline Pu-239 & 0 & 0.0 & 0.1 & 0.1 & 0.1 & 0.1 & 0.1 & Pu-239 & 0 & 0.7 & 1.3 & 1.7 & 1.9 & 2.1 & 2.1 \\
\hline Pu-240 & 0 & 0.0 & 0.0 & 0.0 & 0.0 & 0.0 & 0.0 & Pu-240 & 0 & 0.0 & 0.1 & 0.2 & 0.3 & 0.4 & 0.5 \\
\hline Pu-241 & 0 & 0.0 & 0.0 & 0.0 & 0.0 & 0.0 & 0.0 & Pu-241 & 0 & 0.0 & 0.0 & 0.1 & 0.2 & 0.3 & 0.4 \\
\hline Pu-242 & 0 & 0.0 & 0.0 & 0.0 & 0.0 & 0.0 & 0.0 & Pu-242 & 0 & 0.0 & 0.0 & 0.0 & 0.0 & 0.1 & 0.1 \\
\hline $\mathrm{Pu}$ & 0 & 0.0 & 0.1 & 0.1 & 0.1 & 0.1 & 0.2 & $\mathrm{Pu}$ & 0 & 0.8 & 1.4 & 2.0 & 2.5 & 2.9 & 3.2 \\
\hline Am-241 & 0 & 0.0 & 0.0 & 0.0 & 0.0 & 0.0 & 0.0 & Am-241 & 0 & 0.0 & 0.0 & 0.0 & 0.0 & 0.0 & 0.0 \\
\hline $\mathrm{Am}$ & 0 & 0.0 & 0.0 & 0.0 & 0.0 & 0.0 & 0.0 & Am & 0 & 0.0 & 0.0 & 0.0 & 0.0 & 0.0 & 0.0 \\
\hline
\end{tabular}


Table 7. DIDO Fuel

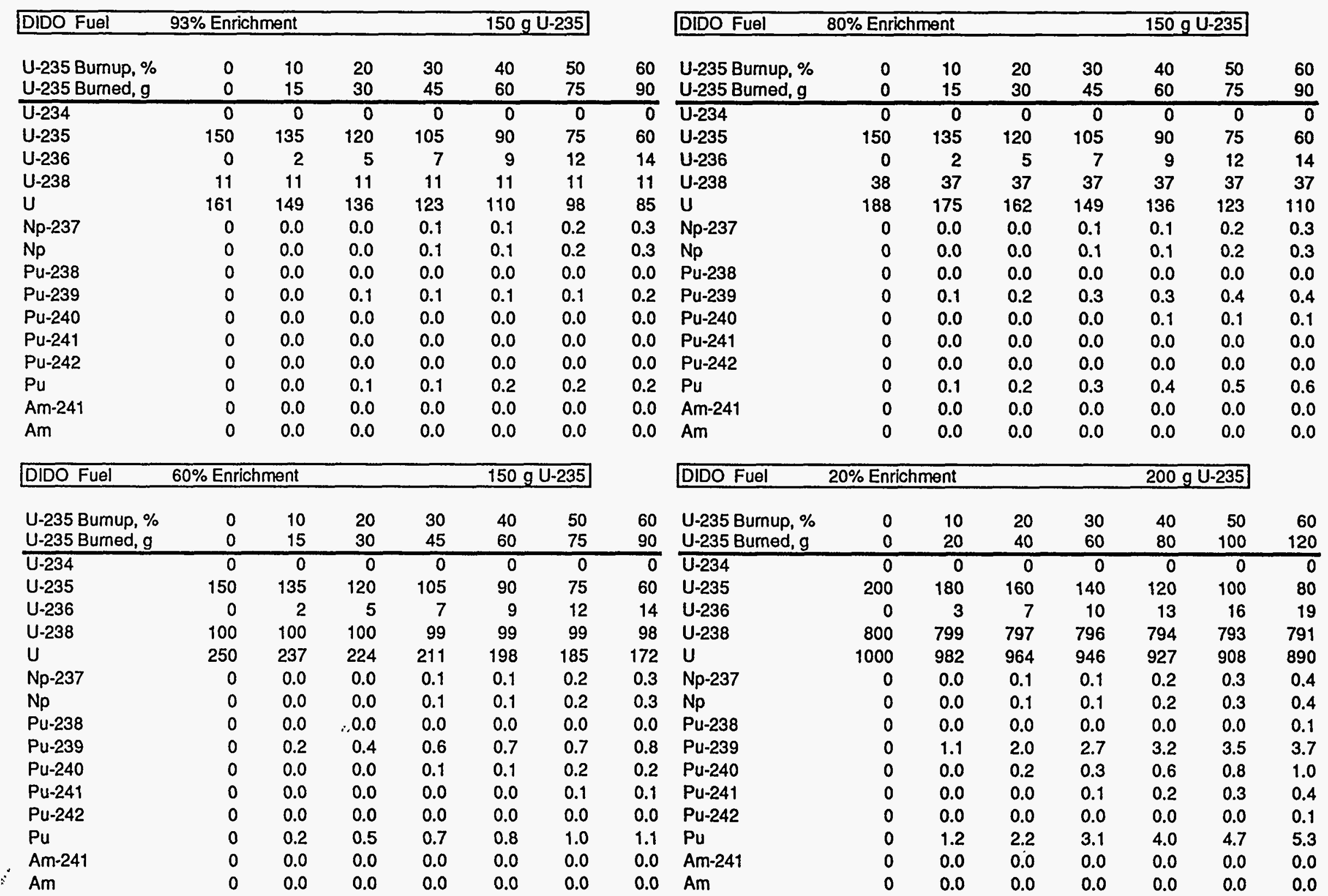




\section{MTR MODEL MASS INVENTORY SIENSITIVITY}

This appendix examines the sensitivity of MTR-type fuel assemblies to the number of fuel plates in the assembly as well as the fuel element specifications for the fuel, clad and coolant. An examination of many MTR-type fuel assemblies shows that the ratio of the coolant channel thickness to the fuel meat thickness, times the number of fuel plates, is nearly a constant. This constant is also proportional to the $\mathrm{H} / \mathrm{U}-235$ atom ratio which can be used to characterize the neutron spectrum in MTR-type fuel assemblies.

Figure A1 shows the $\mathrm{H} / \mathrm{U}-235$ atom ratio as a function of the U-235 mass. The upper curve are for 19 -plate $(0.51 \mathrm{~mm}$ fuel, $0.38 \mathrm{~mm}$ clad, $2.95 \mathrm{~mm}$ coolant $)$ elements and the lower curve are for 23 -plate $(0.51 \mathrm{~mm}$ fuel, $0.38 \mathrm{~mm}$ clad, $2.19 \mathrm{~mm}$ coolant) elements. Most all MTR-type fuel assemblies as a function of the fuel element specifications are within the range $( \pm 6 \%)$ of the average $\mathrm{H} / \mathrm{U}-235$ ratio. 


\section{MTR Fuel Neutron Spectrum Characterization}

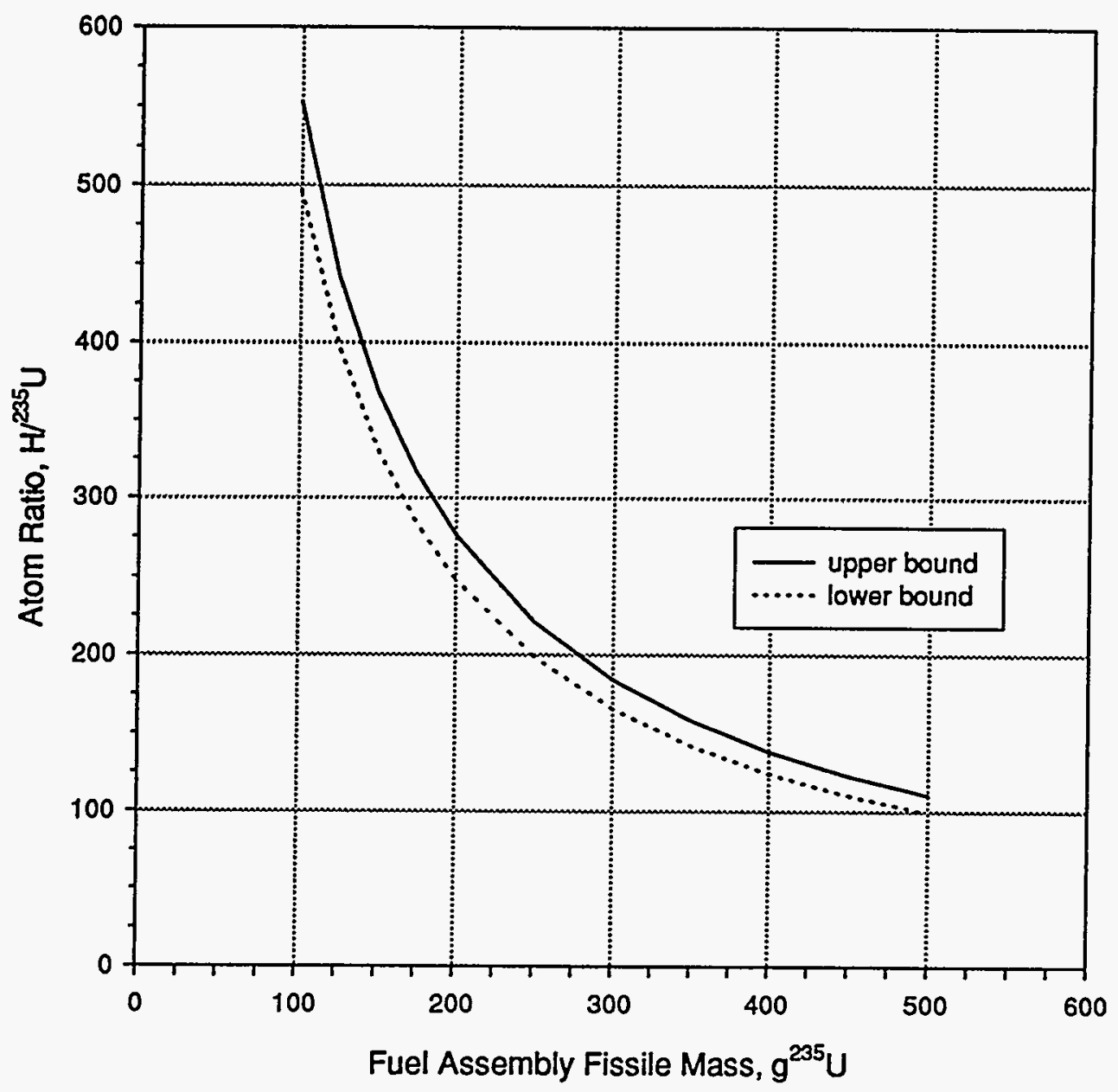

Figure A1. MTR Fuel Assembly Model Sensitivity

Tables A1-A3 show the mass inventory results for MTR fuel assembly types with $300 \mathrm{~g}$ U-235 and 93, 45 and $19.75 \%$ U-235 enrichment. The difference between the upper and lower bound results indicate only small differences in the isotopic masses as a function of fuel element specification. 
Table A1. MTR Fuel 93\% Enrichment

\begin{tabular}{|c|c|c|c|c|c|c|c|c|c|c|}
\hline MTR Upper Bound & \multicolumn{2}{|c|}{$93 \%$ Enrichment } & \multicolumn{3}{|c|}{$300 \mathrm{~g} \mathrm{U}-235$} & \multirow[b]{2}{*}{$\begin{array}{r}40 \\
120\end{array}$} & \multirow[b]{2}{*}{$\begin{array}{r}50 \\
150\end{array}$} & \multirow[b]{2}{*}{$\begin{array}{r}60 \\
180\end{array}$} & \multirow[b]{2}{*}{$\begin{array}{r}70 \\
210 \\
\end{array}$} & \multirow[b]{2}{*}{$\begin{array}{r}80 \\
240\end{array}$} \\
\hline $\begin{array}{l}\text { U-235 Bumup, \% } \\
\text { U-235 Bumed, g }\end{array}$ & $\begin{array}{l}0 \\
0\end{array}$ & $\begin{array}{r}5 \\
15\end{array}$ & $\begin{array}{l}10 \\
30\end{array}$ & $\begin{array}{l}20 \\
60\end{array}$ & $\begin{array}{l}30 \\
90\end{array}$ & & & & & \\
\hline$\overline{U-234}$ & 0 & 0 & 0 & 0 & 0 & 0 & 0 & 0 & 0 & 0 \\
\hline U-235 & 300 & 285 & 270 & 240 & 210 & 180 & 150 & 120 & 90 & 60 \\
\hline$U-236$ & 0 & 3 & 5 & 10 & 15 & 19 & 24 & 28 & 33 & 37 \\
\hline U.238 & 23 & 23 & 22 & 22 & 22 & 22 & 22 & 21 & 21 & 21 \\
\hline $\mathrm{U}$ & 323 & 310 & 297 & 272 & 247 & 221 & 196 & 170 & 144 & 118 \\
\hline $\mathrm{Np}-237$ & 0 & 0.0 & 0.0 & 0.1 & 0.2 & 0.4 & 0.6 & 0.8 & 1.1 & 1.5 \\
\hline $\mathrm{Np}$ & 0 & 0.0 & 0.0 & 0.1 & 0.2 & 0.4 & 0.6 & 0.8 & 1.1 & 1.5 \\
\hline Pu-238 & 0 & 0.0 & 0.0 & 0.0 & 0.0 & 0.0 & 0.1 & 0.1 & 0.2 & 0.3 \\
\hline $\mathrm{Pu}-239$ & 0 & 0.1 & 0.2 & 0.3 & 0.4 & 0.4 & 0.5 & 0.5 & 0.5 & 0.5 \\
\hline $\mathrm{Pu}-240$ & 0 & 0.0 & 0.0 & 0.0 & 0.0 & 0.1 & 0.1 & 0.1 & 0.1 & 0.2 \\
\hline $\mathrm{Pu}-241$ & 0 & 0.0 & 0.0 & 0.0 & 0.0 & 0.0 & 0.0 & 0.1 & 0.1 & 0.1 \\
\hline Pu-242 & 0 & 0.0 & 0.0 & 0.0 & 0.0 & 0.0 & 0.0 & 0.0 & 0.0 & 0.0 \\
\hline $\mathrm{Pu}$ & 0 & 0.1 & 0.2 & 0.3 & 0.4 & 0.5 & 0.7 & 0.8 & 0.9 & 1.1 \\
\hline Am-241 & 0 & 0.0 & 0.0 & 0.0 & 0.0 & 0.0 & 0.0 & 0.0 & 0.0 & 0.0 \\
\hline Am & 0 & 0.0 & 0.0 & 0.0 & 0.0 & 0.0 & 0.0 & 0.0 & 0.0 & 0.0 \\
\hline MTR Lower Bound & \multicolumn{2}{|c|}{$93 \%$ Enrichment } & & \multicolumn{2}{|c|}{$300 \mathrm{~g} \mathrm{U}-235$} & & & & & \\
\hline $\begin{array}{l}\text { U-235 Bumup, \% } \\
\text { U-235 Burned, } g\end{array}$ & $\begin{array}{l}0 \\
0 \\
\end{array}$ & $\begin{array}{r}5 \\
15 \\
\end{array}$ & $\begin{array}{l}10 \\
30 \\
\end{array}$ & $\begin{array}{l}20 \\
60\end{array}$ & $\begin{array}{l}30 \\
90 \\
\end{array}$ & $\begin{array}{r}40 \\
120 \\
\end{array}$ & $\begin{array}{r}50 \\
150 \\
\end{array}$ & $\begin{array}{r}60 \\
180 \\
\end{array}$ & $\begin{array}{r}70 \\
210\end{array}$ & $\begin{array}{r}80 \\
240 \\
\end{array}$ \\
\hline$U-234$ & 0 & 0 & 0 & 0 & 0 & 0 & 0 & 0 & 0 & 0 \\
\hline U-235 & 300 & 285 & 270 & 240 & 210 & 180 & 150 & 120 & 90 & 60 \\
\hline$U-236$ & 0 & 3 & 5 & 10 & 15 & 20 & 24 & 29 & 33 & 37 \\
\hline$U-238$ & 23 & 23 & 22 & 22 & 22 & 22 & 22 & 21 & 21 & 21 \\
\hline U & 323 & 310 & 297 & 272 & 247 & 221 & 196 & 170 & 144 & 118 \\
\hline Np-237 & 0 & 0.0 & 0.0 & 0.1 & 0.2 & 0.4 & 0.6 & 0.9 & 1.2 & 1.6 \\
\hline $\mathrm{Np}$ & 0 & 0.0 & 0.0 & 0.1 & 0.2 & 0.4 & 0.6 & 0.9 & 1.2 & 1.6 \\
\hline Pu-238 & $\therefore 0$ & 0.0 & 0.0 & 0.0 & 0.0 & 0.0 & 0.1 & 0.1 & 0.2 & 0.3 \\
\hline Pu-239 & 0 & 0.1 & 0.2 & 0.3 & 0.4 & 0.4 & 0.5 & 0.5 & 0.5 & 0.5 \\
\hline Pu-240 & 0 & 0.0 & 0.0 & 0.0 & 0.0 & 0.1 & 0.1 & 0.1 & 0.2 & 0.2 \\
\hline Pu-241 & 0 & 0.0 & 0.0 & 0.0 & 0.0 & 0.0 & 0.0 & 0.1 & 0.1 & 0.1 \\
\hline Pu-242 & 0 & 0.0 & 0.0 & 0.0 & 0.0 & 0.0 & 0.0 & 0.0 & 0.0 & 0.0 \\
\hline $\mathrm{Pu}$ & 0 & 0.1 & 0.2 & 0.3 & 0.5 & 0.6 & 0.7 & 0.8 & 1.0 & 1.1 \\
\hline Am-241 & 0 & 0.0 & 0.0 & 0.0 & 0.0 & 0.0 & 0.0 & $0.0^{\circ}$ & 0.0 & 0.0 \\
\hline Am & 0 & 0.0 & 0.0 & 0.0 & 0.0 & 0.0 & 0.0 & 0.0 & 0.0 & 0.0 \\
\hline
\end{tabular}


Table A2. MTR Fuel 45\% Enrichment

\begin{tabular}{|c|c|c|c|c|c|c|c|c|c|c|}
\hline \multirow{2}{*}{$\begin{array}{l}\text { MTR Upper Bound } \\
\text { U-235 Bumup, \% } \\
\text { U-235 Bumed, } 9\end{array}$} & \multicolumn{3}{|c|}{$45 \%$ Enrichment } & \multicolumn{2}{|c|}{$300 \mathrm{~g} \mathrm{U}-235$} & \multirow[b]{2}{*}{$\begin{array}{r}40 \\
120 \\
\end{array}$} & \multirow[b]{2}{*}{$\begin{array}{r}50 \\
150 \\
\end{array}$} & \multirow[b]{2}{*}{$\begin{array}{r}60 \\
180 \\
\end{array}$} & \multirow[b]{2}{*}{$\begin{array}{r}70 \\
210\end{array}$} & \multirow[b]{2}{*}{$\begin{array}{r}80 \\
240 \\
\end{array}$} \\
\hline & $\begin{array}{l}0 \\
0\end{array}$ & $\begin{array}{r}5 \\
15\end{array}$ & $\begin{array}{l}10 \\
30\end{array}$ & $\begin{array}{l}20 \\
60\end{array}$ & $\begin{array}{l}30 \\
90\end{array}$ & & & & & \\
\hline U-234 & 0 & 0 & 0 & 0 & 0 & 0 & 0 & 0 & 0 & 0 \\
\hline U-235 & 300 & 285 & 270 & 240 & 210 & 180 & 150 & 120 & 90 & 60 \\
\hline U-236 & 0 & 3 & 5 & 10 & 15 & 19 & 24 & 29 & 33 & 37 \\
\hline U-238 & 367 & 366 & 365 & 364 & 362 & 361 & 359 & 357 & 355 & 352 \\
\hline$U$ & 667 & 654 & 640 & 614 & 587 & 560 & 533 & 505 & 477 & 449 \\
\hline Np-237 & 0 & 0.0 & 0.0 & 0.1 & 0.2 & 0.4 & 0.6 & 0.9 & 1.2 & 1.5 \\
\hline $\mathrm{Np}$ & 0 & 0.0 & 0.0 & 0.1 & 0.2 & 0.4 & 0.6 & 0.9 & 1.2 & 1.5 \\
\hline Pu-238 & 0 & 0.0 & 0.0 & 0.0 & 0.0 & 0.0 & 0.1 & 0.1 & 0.2 & 0.3 \\
\hline Pu-239 & 0 & 0.6 & 1.2 & 2.2 & 2.9 & 3.4 & 3.8 & 3.9 & 3.8 & 3.6 \\
\hline Pu-240 & 0 & 0.0 & 0.0 & 0.2 & 0.3 & 0.6 & 0.8 & 1.0 & 1.2 & 1.4 \\
\hline Pu-241 & 0 & 0.0 & 0.0 & 0.0 & 0.1 & 0.2 & 0.3 & 0.5 & 0.6 & 0.7 \\
\hline Pu-242 & 0 & 0.0 & 0.0 & 0.0 & 0.0 & 0.0 & 0.0 & 0.1 & 0.2 & 0.3 \\
\hline $\mathrm{Pu}$ & 0 & 0.6 & 1.3 & 2.4 & 3.4 & 4.2 & 5.0 & 5.6 & 6.0 & 6.3 \\
\hline Am-241 & 0 & 0.0 & 0.0 & 0.0 & 0.0 & 0.0 & 0.0 & 0.0 & 0.0 & 0.0 \\
\hline Am & 0 & 0.0 & 0.0 & 0.0 & 0.0 & 0.0 & 0.0 & 0.0 & 0.0 & 0.0 \\
\hline MTR Lower Bound & \multicolumn{2}{|c|}{$45 \%$ Enrichment } & & \multicolumn{2}{|c|}{$300 \mathrm{~g} \mathrm{U}-235$} & & & & & \\
\hline $\begin{array}{l}\text { U-235 Bumup, \% } \\
\text { U-235 Burned, g }\end{array}$ & $\begin{array}{l}0 \\
0 \\
\end{array}$ & $\begin{array}{r}5 \\
15 \\
\end{array}$ & $\begin{array}{l}10 \\
30 \\
\end{array}$ & $\begin{array}{l}20 \\
60 \\
\end{array}$ & $\begin{array}{l}30 \\
90 \\
\end{array}$ & $\begin{array}{r}40 \\
120 \\
\end{array}$ & $\begin{array}{r}50 \\
150 \\
\end{array}$ & $\begin{array}{r}60 \\
180 \\
\end{array}$ & $\begin{array}{r}70 \\
210 \\
\end{array}$ & $\begin{array}{r}80 \\
240 \\
\end{array}$ \\
\hline$U-234$ & 0 & 0 & 0 & 0 & 0 & 0 & 0 & 0 & 0 & 0 \\
\hline U-235 & 300 & 285 & 270 & 240 & 210 & 180 & 150 & 120 & 90 & 60 \\
\hline U-236 & 0 & 3 & 5 & 10 & 15 & 20 & 24 & 29 & 33 & 37 \\
\hline$U-238$ & 367 & 366 & 365 & 364 & 362 & 360 & 358 & 356 & 354 & 351 \\
\hline$U$ & 667 & 654 & 640 & 614 & 587 & 560 & 532 & 505 & 477 & 448 \\
\hline $\mathrm{Np}-237$ & 0 & 0.0 & 0.0 & 0.1 & 0.2 & 0.4 & 0.7 & 0.9 & 1.3 & 1.6. \\
\hline $\mathrm{Np}$ & 0 & 0.0 & 0.0 & 0.1 & 0.2 & 0.4 & 0.7 & 0.9 & 1.3 & 1.6 \\
\hline Pu-238 & $\therefore 0$ & 0.0 & 0.0 & 0.0 & 0.0 & 0.0 & 0.1 & 0.1 & 0.2 & 0.4 \\
\hline Pu-239 & 0 & 0.7 & 1.3 & 2.3 & 3.1 & 3.7 & 4.0 & 4.1 & 4.0 & 3.8 \\
\hline Pu-240 & 0 & 0.0 & 0.1 & 0.2 & 0.4 & 0.6 & 0.8 & 1.0 & 1.3 & 1.4 \\
\hline Pu-241 & 0 & 0.0 & 0.0 & 0.0 & 0.1 & 0.2 & 0.4 & 0.5 & 0.7 & 0.8 \\
\hline Pu-242 & 0 & 0.0 & 0.0 & 0.0 & 0.0 & 0.0 & 0.1 & 0.1 & 0.2 & 0.4 \\
\hline $\mathrm{Pu}$ & 0 & 0.7 & 1.4 & 2.6 & 3.6 & $4.5^{\prime}$ & 5.3 & 5.9 & 6.4 & 6.7 \\
\hline Am-241 & 0 & 0.0 & 0.0 & 0.0 & 0.0 & 0.0 & 0.0 & 0.0 & 0.0 & 0.0 \\
\hline Am & 0 & 0.0 & 0.0 & 0.0 & 0.0 & 0.0 & 0.0 & 0.0 & 0.0 & 0.0 \\
\hline
\end{tabular}


Table A3. MTR Fuel 19.75\% Enrichment

\begin{tabular}{|c|c|c|c|c|c|c|c|c|c|c|}
\hline MTR Upper Bound & $19.75 \%$ & hment & & 300 & 35 & & & & & \\
\hline $\begin{array}{l}\text { U-235 Burnup, \% } \\
\text { U-235 Bumed, g }\end{array}$ & $\begin{array}{l}0 \\
0\end{array}$ & $\begin{array}{r}5 \\
15\end{array}$ & $\begin{array}{l}10 \\
30\end{array}$ & $\begin{array}{l}20 \\
60\end{array}$ & $\begin{array}{l}30 \\
90\end{array}$ & $\begin{array}{r}40 \\
120\end{array}$ & $\begin{array}{r}50 \\
150\end{array}$ & $\begin{array}{r}60 \\
180\end{array}$ & $\begin{array}{r}70 \\
210\end{array}$ & $\begin{array}{r}80 \\
240\end{array}$ \\
\hline U-234 & 0 & 0 & $\overline{0}$ & 0 & 0 & 0 & 0 & 0 & 0 & 0 \\
\hline U-235 & 300 & 285 & 270 & 240 & 210 & 180 & 150 & 120 & 90 & 60 \\
\hline U-236 & 0 & 3 & 5 & 10 & 15 & 20 & 24 & 29 & 33 & 37 \\
\hline U-238 & 1219 & 1218 & 1216 & 1213 & 1209 & 1205 & 1201 & 1197 & 1191 & 1184 \\
\hline $\mathrm{U}$ & 1519 & 1505 & 1491 & 1463 & 1434 & 1405 & 1375 & 1345 & 1314 & 1281 \\
\hline $\mathrm{Np}-237$ & 0 & 0.0 & 0.0 & 0.1 & 0.2 & 0.4 & 0.6 & 0.9 & 1.2 & 1.6 \\
\hline $\mathrm{Np}$ & 0 & 0.0 & 0.0 & 0.1 & 0.2 & 0.4 & 0.6 & 0.9 & 1.2 & 1.6 \\
\hline Pu-238 & 0 & 0.0 & 0.0 & 0.0 & 0.0 & 0.0 & 0.1 & 0.1 & 0.2 & 0.3 \\
\hline Pu-239 & 0 & 1.3 & 2.6 & 4.7 & 6.3 & 7.5 & 8.3 & 8.7 & 8.7 & 8.4 \\
\hline Pu-240 & 0 & 0.0 & 0.1 & 0.4 & 0.7 & 1.2 & 1.7 & 2.2 & 2.7 & 3.2 \\
\hline Pu-241 & 0 & 0.0 & 0.0 & 0.1 & 0.2 & 0.4 & 0.7 & 1.0 & 1.4 & 1.6 \\
\hline Pu-242 & 0 & 0.0 & 0.0 & 0.0 & 0.0 & 0.0 & 0.1 & 0.2 & 0.4 & 0.7 \\
\hline $\mathrm{Pu}$ & 0 & 1.4 & 2.7 & 5.1 & 7.3 & 9.2 & 10.9 & 12.3 & 13.4 & 14.3 \\
\hline Am-241 & 0 & 0.0 & 0.0 & 0.0 & 0.0 & 0.0 & 0.0 & 0.0 & 0.0 & 0.1 \\
\hline Am & 0 & 0.0 & 0.0 & 0.0 & 0.0 & 0.0 & 0.0 & 0.0 & 0.0 & 0.1 \\
\hline
\end{tabular}

\begin{tabular}{|ll}
\hline MTR Lower Bound $\quad 19.75 \%$ Enrichment & $300 \mathrm{~g} \mathrm{U}-235$ \\
\hline
\end{tabular}

\begin{tabular}{|c|c|c|c|c|c|c|c|c|c|c|}
\hline $\begin{array}{l}\text { U-235 Burnup, \% } \\
\text { U-235 Burned, g }\end{array}$ & $\begin{array}{l}0 \\
0\end{array}$ & $\begin{array}{r}5 \\
15\end{array}$ & $\begin{array}{l}10 \\
30\end{array}$ & $\begin{array}{l}20 \\
60\end{array}$ & $\begin{array}{l}30 \\
90\end{array}$ & $\begin{array}{r}40 \\
120 \\
\end{array}$ & $\begin{array}{r}50 \\
150\end{array}$ & $\begin{array}{r}60 \\
180\end{array}$ & $\begin{array}{r}70 \\
210\end{array}$ & $\begin{array}{r}80 \\
240 \\
\end{array}$ \\
\hline U-234 & 0 & 0 & 0 & 0 & 0 & 0 & 0 & 0 & 0 & 0 \\
\hline U-235 & 300 & 285 & 270 & 240 & 210 & 180 & 150 & 120 & 90 & 60 \\
\hline U-236 & 0 & 3 & 5 & 10 & 15 & 20 & 24 & 29 & 33 & 37 \\
\hline U-238 & 1219 & 1217 & 1216 & 1212 & 1209 & 1205 & 1200 & 1195 & 1189 & 1182 \\
\hline U & 1519 & 1505 & 1491 & 1462 & 1433 & 1404 & 1374 & 1344 & 1312 & 1279 \\
\hline Np-237 & 0 & 0.0 & 0.0 & 0.1 & 0.2 & 0.4 & 0.7 & 1.0 & 1.3 & 1.8 \\
\hline $\mathrm{Np}$ & 0 & 0.0 & 0.0 & 0.1 & 0.2 & 0.4 & 0.7 & 1.0 & 1.3 & 1.8 \\
\hline Pu-238 & $\therefore \quad 0$ & 0.0 & 0.0 & 0.0 & 0.0 & 0.0 & 0.1 & 0.1 & 0.2 & 0.4 \\
\hline$P u-239$ & 0 & 1.4 & 2.8 & 5.0 & 6.7 & 8.0 & 8.8 & 9.2 & 9.2 & 8.8 \\
\hline Pu-240 & 0 & 0.0 & 0.1 & 0.4 & 0.8 & 1.3 & 1.8 & 2.3 & 2.8 & 3.3 \\
\hline Pu-241 & 0 & 0.0 & 0.0 & 0.1 & 0.2 & 0.5 & 0.8 & 1.2 & 1.5 & 1.8 \\
\hline Pu-242 & 0 & 0.0 & 0.0 & 0.0 & 0.0 & 0.0 & 0.1 & 0.2 & 0.5 & 0.8 \\
\hline $\mathrm{Pu}$ & 0 & 1.5 & 2.9 & 5.5 & 7.8 & 9.8 & 11.6 & 13.1 & 14.3 & 15.1 \\
\hline Am-241 & 0 & 0.0 & 0.0 & 0.0 & 0.0 & 0.0 & 0.0 & 0.0 & 0.0 & 0.1 \\
\hline Am & 0 & 0.0 & 0.0 & 0.0 & 0.0 & 0.0 & 0.0 & 0.0 & 0.0 & 0.1 \\
\hline
\end{tabular}




\section{APPENDIX B}

\section{U-234 AND U-236 MASS INVENTORY SENSITIVITY}

The initial fuel composition of some reactor fuels may contain specifications for U-234 and/or U-236 in addition to the usual specifications for U-235 and U-238. It is the purpose of this appendix to evaluate the effect that U-234 and U-236 have on the overall fuel assembly mass inventory when these isotopes are or are not included in the initial fuel assembly composition.

A comparison of the fuel mass inventory for a HEU and a LEU fuel composition, with and without initial enrichments of U-234 and U-236, are shown in Table B1.

Typical enrichments of U-234 and U-236 in research reactor fuels are less than 1\%; these specific data are for typical TRIGA fuel compositions.

The upper section of Table B1 shows the mass inventory for HEU and LEU fuels with initial enrichments of U-234 and U-236, and the lower section shows similar data for the same fuels but without initial U-234 and U-236 enrichment. The result of this comparison shows that to first-order, any initial mass of U-234 or U-236 can be simply added to the mass inventory for U-234, U-236 and total $U$ at any burnup level. The mass inventory for $\mathrm{Np}-237$ and $\mathrm{Pu}-238$ which are also functions of the U-236 mass, are not substantially affected by an initial enrichment of U-236. 
Table B1. TRIGA Fuel 25-Rod Cluster Model

\begin{tabular}{|c|c|c|c|c|c|c|c|c|c|c|c|c|c|c|c|}
\hline \multirow{2}{*}{$\begin{array}{l}\text { TRIGA Fuel } \\
\text { U-235 Burnup, \% } \\
\text { U-235 Bumed, g }\end{array}$} & \multicolumn{3}{|c|}{$10 w t \%$ U, 93.1\% Enrichment } & \multicolumn{3}{|c|}{$41.4 \mathrm{~g} \mathrm{U}-235$} & \multirow[b]{2}{*}{$\begin{array}{r}60 \\
24.8 \\
\end{array}$} & \multirow{2}{*}{$\begin{array}{l}\text { TRIGA Fuel } \\
\text { U-235 Bumup, \% } \\
\text { U-235 Bumed, } g\end{array}$} & \multicolumn{3}{|c|}{$45 w t \%$ U, 19.7\% Enrichment } & \multicolumn{3}{|c|}{$53.6 \mathrm{~g} \mathrm{U}-235$} & \multirow[b]{2}{*}{$\begin{array}{r}60 \\
32.2 \\
\end{array}$} \\
\hline & $\begin{array}{r}0 \\
0.0\end{array}$ & $\begin{array}{r}10 \\
4.1 \\
\end{array}$ & $\begin{array}{r}20 \\
8.3 \\
\end{array}$ & $\begin{array}{r}30 \\
12.4 \\
\end{array}$ & $\begin{array}{r}40 \\
16.6 \\
\end{array}$ & $\begin{array}{r}50 \\
20.7\end{array}$ & & & $\begin{array}{r}0 \\
0.0\end{array}$ & $\begin{array}{r}10 \\
5.4 \\
\end{array}$ & $\begin{array}{r}20 \\
10.7\end{array}$ & $\begin{array}{r}30 \\
16.1 \\
\end{array}$ & $\begin{array}{r}40 \\
21.4\end{array}$ & $\begin{array}{r}50 \\
26.8\end{array}$ & \\
\hline U-234 & 0.4 & 0.4 & 0.4 & 0.4 & 0.4 & 0.3 & 0.3 & U-234 & 0.4 & 0.4 & 0.4 & 0.3 & 0.3 & 0.3 & 0.3 \\
\hline U-235 & 41.4 & 37.2 & 33.1 & 29.0 & 24.8 & 20.7 & 16.6 & U-235 & 53.6 & 48.3 & 42.9 & 37.5 & 32.2 & 26.8 & 21.4 \\
\hline U-236 & 0.2 & 1.0 & 1.7 & 2.4 & 3.1 & 3.8 & 4.4 & U-236 & 0.7 & 1.7 & 2.7 & 3.7 & 4.6 & 5.5 & 6.4 \\
\hline U-238 & 2.4 & 2.4 & 2.4 & 2.3 & 2.3 & 2.2 & 2.2 & U-238 & 217.4 & 216.5 & 215.6 & 214.6 & 213.5 & 212.3 & 210.9 \\
\hline $\mathbf{U}$ & 44.5 & 41.0 & 37.6 & 34.1 & 30.6 & 27.1 & 23.5 & $\mathbf{U}$ & 272.1 & 266.9 & 261.6 & 256.1 & 250.6 & 244.9 & 239.0 \\
\hline Np-237 & 0 & 0.0 & 0.0 & 0.1 & 0.1 & 0.2 & 0.2 & Np-237 & 0 & 0.0 & 0.1 & 0.1 & 0.2 & 0.3 & 0.4 \\
\hline Np & 0 & 0.0 & 0.0 & 0.1 & 0.1 & 0.2 & 0.2 & $N p$ & 0 & 0.0 & 0.1 & 0.1 & 0.2 & 0.3 & 0.4 \\
\hline Pu-238 & 0 & 0.0 & 0.0 & 0.0 & 0.0 & 0.0 & 0.0 & Pu-238 & 0 & 0.0 & 0.0 & 0.0 & 0.0 & 0.1 & 0.1 \\
\hline Pu-239 & 0 & 0.0 & 0.1 & 0.1 & 0.1 & 0.1 & 0.1 & Pu-239 & 0 & 0.7 & 1.3 & 1.7 & 1.9 & 2.1 & 2.1 \\
\hline Pu-240 & 0 & 0.0 & 0.0 & 0.0 & 0.0 & 0.0 & 0.0 & Pu-240 & 0 & 0.0 & 0.1 & 0.2 & 0.3 & 0.4 & 0.5 \\
\hline Pu-241 & 0 & 0.0 & 0.0 & 0.0 & 0.0 & 0.0 & 0.0 & Pu-241 & 0 & 0.0 & 0.0 & 0.1 & 0.2 & 0.3 & 0.4 \\
\hline Pu-242 & 0 & 0.0 & 0.0 & 0.0 & 0.0 & 0.0 & 0.0 & Pu-242 & 0 & 0.0 & 0.0 & 0.0 & 0.0 & 0.1 & 0.1 \\
\hline $\mathrm{Pu}$ & 0 & 0.0 & 0.1 & 0.1 & 0.1 & 0.1 & 0.2 & $\mathrm{Pu}$ & 0 & 0.8 & 1.4 & 2.0 & 2.5 & 2.9 & 3.2 \\
\hline Am-241 & 0 & 0.0 & 0.0 & 0.0 & 0.0 & 0.0 & 0.0 & Am-241 & 0 & 0.0 & 0.0 & 0.0 & 0.0 & 0.0 & 0.0 \\
\hline Am & 0 & 0.0 & 0.0 & 0.0 & 0.0 & 0.0 & 0.0 & Am & 0 & 0.0 & 0.0 & 0.0 & 0.0 & 0.0 & 0.0 \\
\hline TRIGA Fuel & $10 w t \% U_{1}$ & $3.1 \% \mathrm{E}$ & chment & & 41.4 & -235 & & TRIGA Fuel & $45 w t \% U$ & $19.7 \% \mathrm{E}$ & richment & & 53.6 & U-235 & \\
\hline $\begin{array}{l}\text { U-235 Bumup, \% } \\
\text { U-235 Burned, g }\end{array}$ & $\begin{array}{r}0 \\
0.0 \\
\end{array}$ & $\begin{array}{r}10 \\
4.1 \\
\end{array}$ & $\begin{array}{r}20 \\
8.3 \\
\end{array}$ & $\begin{array}{r}30 \\
12.4 \\
\end{array}$ & $\begin{array}{r}40 \\
16.6 \\
\end{array}$ & $\begin{array}{r}50 \\
20.7 \\
\end{array}$ & $\begin{array}{r}60 \\
24.8 \\
\end{array}$ & $\begin{array}{l}\text { U-235 Bumup, \% } \\
\text { U-235 Bumed, g }\end{array}$ & $\begin{array}{r}0 \\
0.0 \\
\end{array}$ & $\begin{array}{r}10 \\
5.4 \\
\end{array}$ & $\begin{array}{r}20 \\
10.7 \\
\end{array}$ & $\begin{array}{r}30 \\
16.1 \\
\end{array}$ & $\begin{array}{r}40 \\
21.4 \\
\end{array}$ & $\begin{array}{r}50 \\
26.8 \\
\end{array}$ & $\begin{array}{r}60 \\
32.2 \\
\end{array}$ \\
\hline U-234 & 0.0 & 0.0 & 0.0 & 0.0 & 0.0 & 0.0 & 0.0 & U-234 & 0.0 & 0.0 & 0.0 & 0.0 & 0.0 & 0.0 & 0.0 \\
\hline U-235 & 41.4 & 37.2 & 33.1 & 29.0 & 24.8 & 20.7 & 16.6 & U-235 & 53.6 & 48.3 & 42.9 & 37.5 & 32.2 & 26.8 & 21.4 \\
\hline U-236 & 0.0 & 0.8 & 1.5 & 2.3 & 2.9 & 3.6 & 4.2 & U-236 & 0.0 & 1.1 & 2.1 & 3.1 & 4.0 & 4.9 & 5.7 \\
\hline U-238 & 2.4 & 2.4 & 2.4 & 2.3 & 2.3 & 2.2 & 2.2 & U-238 & 217.4 & 216.5 & 215.6 & 214.6 & 213.5 & 212.3 & 211.0 \\
\hline $\mathrm{U}$ & 43.8 & 40.4 & 37.0 & 33.5 & 30.1 & 26.5 & 23.0 & $\mathbf{U}$ & 271.1 & 265.9 & 260.6 & 255.2 & 249.7 & 244.0 & 238.1 \\
\hline Np-237 & 0 & 0.0 & 0.0 & 0.1 & 0.1 & 0.1 & 0.2 & Np-237 & 0 & 0.0 & 0.0 & 0.1 & 0.2 & 0.2 & 0.3 \\
\hline $\mathrm{Np}$ & 0 & 0.0 & 0.0 & 0.1 & 0.1 & 0.1 & 0.2 & Np & 0 & 0.0 & 0.0 & 0.1 & 0.2 & 0.2 & 0.3 \\
\hline Pu-238 & 0 & 0.0 & 0,0 & 0.0 & 0.0 & 0.0 & 0.0 & Pu-238 & 0 & 0.0 & 0.0 & 0.0 & 0.0 & 0.0 & 0.1 \\
\hline Pu-239 & 0 & 0.0 & 0.1 & 0.1 & 0.1 & 0.1 & 0.1 & Pu-239 & 0 & 0.7 & 1.3 & 1.7 & 1.9 & 2.1 & 2.1 \\
\hline Pu-240 & 0 & 0.0 & 0.0 & 0.0 & 0.0 & 0.0 & 0.0 & Pu-240 & 0 & 0.0 & 0.1 & 0.2 & 0.3 & 0.4 & 0.5 \\
\hline Pu-241 & 0 & 0.0 & 0.0 & 0.0 & 0.0 & 0.0 & 0.0 & Pu-241 & 0 & 0.0 & 0.0 & 0.1 & 0.2 & 0.3 & 0.4 \\
\hline Pu-242 & 0 & 0.0 & 0.0 & 0.0 & 0.0 & 0.0 & 0.0 & Pu-242 & 0 & 0.0 & 0.0 & 0.0 & 0.0 & 0.1 & 0.1 \\
\hline $\mathrm{Pu}$ & 0 & 0.0 & 0.1 & 0.1 & 0.1 & 0.1 & 0.2 & $\mathrm{Pu}$ & 0 & 0.8 & 1.4 & 2.0 & 2.5 & 2.9 & 3.2 \\
\hline$A m-241$ & 0 & 0.0 & 0.0 & 0.0 & 0.0 & 0.0 & 0.0 & Am-241 & 0 & 0.0 & 0.0 & 0.0 & 0.0 & 0.0 & 0.0 \\
\hline Am & 0 & 0.0 & 0.0 & 0.0 & 0.0 & 0.0 & 0.0 & $\mathrm{Am}$ & 0 & 0.0 & 0.0 & 0.0 & 0.0 & 0.0 & 0.0 \\
\hline
\end{tabular}

